\title{
Cis-acting super-enhancer IncRNAs as biomarkers to early-stage breast cancer
}

\author{
Ali S. Ropri ${ }^{1 *} \mathbb{D}$, Rebecca S. DeVaux ${ }^{1}$, Jonah Eng ${ }^{2}$, Sridar V. Chittur ${ }^{1,3}$ and Jason I. Herschkowitz ${ }^{1}$
}

\begin{abstract}
Background: Increased breast cancer screening over the past four decades has led to a substantial rise in the diagnosis of ductal carcinoma in situ (DCIS). Although DCIS lesions precede invasive ductal carcinoma (IDC), they do not always transform into cancer. The current standard-of-care for DCIS is an aggressive course of therapy to prevent invasive and metastatic disease resulting in over-diagnosis and over-treatment. Thus, there is a critical need to identify functional determinants of progression of DCIS to IDC to allow discrimination between indolent and aggressive disease. Recent studies show that super-enhancers, in addition to promoting other gene transcription, are themselves transcribed producing super-enhancer associated long noncoding RNAs (SE-IncRNAs). These SE-IncRNAs can interact with their associated enhancer regions in cis and influence activities and expression of neighboring genes. Furthermore, they represent a novel, untapped group of therapeutic targets.
\end{abstract}

Methods: With an integrative analysis of enhancer loci with global expression of SE-IncRNAs in the MCF10A progression series, we have identified differentially expressed SE-InCRNAs which can identify mechanisms for DCIS to IDC progression. Furthermore, cross-referencing these SE-IncRNAs with patient samples in the The Cancer Genome Atlas (TCGA) database, we have unveiled 27 clinically relevant SE-IncRNAs that potentially interact with their enhancer to regulate nearby gene expression. To complement SE-IncRNA expression studies, we conducted an unbiased global analysis of super-enhancers that are acquired or lost in progression.

Results: Here we designate SE-IncRNAs RP11-379F4.4 and RP11-465B22.8 as potential markers of progression of DCIS to IDC through regulation of the expression of their neighboring genes (RARRES1 and miR-200b, respectively). Moreover, we classified 403 super-enhancer regions in MCF10A normal cells, 627 in AT1, 1053 in DCIS, and 320 in CA1 cells. Comparison analysis of acquired/lost super-enhancer regions with super-enhancer regions classified in 47 ER positive patients, 10 triple negative breast cancer (TNBC) patients, and 11 TNBC cell lines reveal critically acquired pathways including STAT signaling and NF-kB signaling. In contrast, protein folding, and local estrogen production are identified as major pathways lost in progression.

Conclusion: Collectively, these analyses identify differentially expressed SE-IncRNAs and acquired/lost super-enhancers in progression of breast cancer important for promoting DCIS lesions to IDC.

Keywords: Breast cancer progression, Ductal carcinoma in situ, Super-enhancer long non-coding RNAs, Superenhancers

*Correspondence: aropri@albany.edu

1 Department of Biomedical Sciences, Cancer Research Center, University at Albany, 1 Discovery Drive, Suite 317, Rensselaer, NY 12144, USA

Full list of author information is available at the end of the article

\section{Background}

Breast cancer can be defined as a group of diseases with heterogeneous origins, molecular profiles and behaviors characterized by uncontrolled proliferation of cells within the mammary gland. Around one in eight women in the USA will develop breast cancer in their lifetime, making original author(s) and the source, provide a link to the Creative Commons licence, and indicate if changes were made. The images or other third party material in this article are included in the article's Creative Commons licence, unless indicated otherwise in a credit line to the material. If material is not included in the article's Creative Commons licence and your intended use is not permitted by statutory regulation or exceeds the permitted use, you will need to obtain permission directly from the copyright holder. To view a copy of this licence, visit http://creativecommons.org/licenses/by/4.0/. The Creative Commons Public Domain Dedication waiver (http://creativeco mmons.org/publicdomain/zero/1.0/) applies to the data made available in this article, unless otherwise stated in a credit line to the data. 
it the second most frequently diagnosed cancer behind skin cancer [1]. In 2021, an estimated 281,550 cases of invasive breast carcinoma are predicted to be diagnosed, and over 40,000 deaths are expected, accounting for almost $7 \%$ of all cancer mortality each year. Ductal carcinoma in situ (DCIS) is the presence of abnormal cells inside a milk duct in the breast and is a precursor to invasive cancer. DCIS accounts for $20 \%$ of breast cancer diagnoses per year [2], however, while not all DCIS lesions progress to invasive cancer, all are treated as such leading to overdiagnosis and overtreatment. In fact, DCIS lesions sometimes grow so slowly that even without treatment it would not affect a woman's health. Long-term studies have found that only $40 \%$ of women with untreated DCIS are ultimately diagnosed with invasive breast cancer [3]. The steep increase in diagnosis of DCIS over the past $30-40$ years is believed to be a result of more frequent mammography [4]. However, because over half of these in situ lesions will not progress to invasive breast cancer, controversies have arisen about approaches to treatment.

As early screening is advocated and on the rise, better understanding of the progression of non-invasive to invasive breast cancer is a prerequisite for correct diagnosis of patients. There is a need to highlight functional determinants DCIS progression to invasive ductal carcinoma (IDC) thus allowing discrimination between indolent and potentially metastatic breast cancers. Understanding the mechanisms of transition of normal breast to invasive breast cancer can have significant implications for preventive and clinical management of breast cancer.

Transcriptome reprogramming is one of the crucial characteristics of cancer, where aberrant gene expression promotes tumor initiation, progression, and metastasis. This can be amplified by cis-element changes in noncoding genomic regions [5]. For example, super-enhancers (SEs), also known as stretch enhancers, are genomic regions where multiple enhancers are clustered together. They exert more potent effects than typical enhancers, are characterized by high levels of Mediator binding, and are associated primarily with tissue-specific genes [6]. Super-enhancers are most likely the major contributors to the expression of their associated genes [7]. There have also been several indications of links between superenhancers and diseases [8]. Recent studies have shown that SEs play key roles in determining cell identity in both healthy and pathological states. Over 25,000 enhancers were identified as differentially activated in renal, breast, and prostate tumor cells, as compared with normal cells. This suggests a network between malignancy and enhancer activity [9]. Likewise, cancer cells have been shown to acquire super-enhancers at oncogenes and cancerous phenotype relies on the abnormal transcription propelled by Ses [10]. Additionally, super-enhancer regions are transcribed generating long non-coding RNAs (lncRNAs) that may play a pivotal role in assisting the super-enhancer function [11].

Long non-coding RNAs are a large class of non-coding transcripts that are $>200$ nucleotides in length and do not encode proteins [12]. Evidence has indicated that lncRNAs regulate gene expression at the levels of epigenetic modification, transcription, translation, and posttranslation [13]. At the transcription level, these RNAs can be associated with super-enhancer regions, and interact with enhancer sequences to influence activities of neighboring genes. A study published in 2011 shows that enhancer associated lncRNA (e-lncRNA) HOTTIP, which resides at the $5^{\prime}$ tip of the HOXA locus, regulates the transcription of various HOXA genes in vivo through chromosomal looping of its enhancer to the promoter region of these genes [14]. In the same fashion, Sigova et al. observe that nascent e-lncRNAs are necessary for the recruitment of Yin-Yang-1 transcription factor to its target enhancer [15]. As a result, e-lncRNAs have been indicated to play a vital role in engaging with transcription factors and localizing them to cognate enhancers. Enhancer lncRNAs are also involved in diverse tumor biological processes, including cell proliferation, apoptosis, invasion, metastasis, and angiogenesis as well as interacting with their enhancers to regulate genes specific to cell identity [16].

Previously, our laboratory has profiled global lncRNA expression in a unique patient-based model of breast cancer progression, wherein early DCIS lesions are directly contiguous with an IDC lesion. From this unbiased patient-based model, 132 lncRNAs were identified as differentially expressed in early breast cancer progression [17], of which 78 were transcribed from superenhancer regions. This statistically significant enrichment in enhancer associated lncRNAs suggests a core mechanism of breast cancer progression. In this study, we identify super-enhancer associated lncRNAs (SE-lncRNAs) that are differentially expressed between non-invasive and invasive breast cancer in the MCF10A progression series as well as DCIS and IDC patient samples. We also designate two of the most promising SE-lncRNAs from our list for their potential cis-acting capabilities in regulating nearby gene expression crucial for progression to IDC. Furthermore, we highlight super-enhancers that are acquired or lost in progression to IDC, giving insight about genes and pathways these super-enhancers regulate which may be necessary for progression.

\section{Materials and methods}

\section{Cell culture}

The MCF10A progression series (MCF10A, MCF10AAT1 (AT1), MCF10A-DCIS (DCIS or DCIS.com), and 
MCF10A-CA1 (CA1)) were purchased from the Barbara Ann Karmanos Cancer Institute and maintained in a culture of Dulbecco's modified Eagle's medium (DMEM) medium supplemented with $5 \%$ horse serum, $20 \mathrm{ng} /$ $\mathrm{mL}$ EGF, $0.5 \mathrm{mg} / \mathrm{mL}$ hydrocortisone, $100 \mathrm{ng} / \mathrm{mL}$ cholera toxin, $10 \mu \mathrm{g} / \mathrm{mL}$ insulin, and $1 \times$ antibiotic-antimycotic (Gibco, Grand Island, NY). All cell lines were verified by STR analysis and routinely screened for mycoplasma contamination.

\section{Microarray}

Arraystar Super-enhancer lncRNA arrays were used to systematically profile 7753 lncRNAs transcribed from super-enhancer (SE) regions along with 7040 corresponding SE-regulated protein coding genes. Briefly, an optimized mixture of oligo(dT) and random primers, each containing a T7 polymerase promoter, is annealed to the RNA. The cDNA is synthesized by reverse transcription followed by 5' adapter annealing and PCR amplification. Finally, cyanine 3- or cyanine 5-labeled cRNA is synthesized by in vitro transcription from the $\mathrm{T} 7$ promoter by T7 RNA polymerase.

\section{Gene expression analysis}

Gene expression was confirmed for the 27 potentially cis-acting SE-lncRNAs and 4 highest differentiated SElncRNAs. Forty-eight hours after seeding, total RNA was collected using Omega Bio-Tek E.Z.N.A. Total RNA Kit (Catalog No. R6-834-02). RNA was subsequently reversetranscribed using Applied Biosystems High-Capacity cDNA Reverse Transcription Kit (Life Technologies Catalog No. 4368813) and analyzed by quantitative reverse transcription PCR (qRT-PCR) with Sybr Green on the QuantStudio $12 \mathrm{~K}$ Flex (Thermo Fisher Scientific) for indicated genes. The $\Delta \Delta C \mathrm{C}$ method was used to determine gene expression fold change with $18 \mathrm{~s}$, Act-b, and GAPDH used for controls.

\section{Cellular localization}

Whole cell lysate, cytoplasmic fraction, and nuclear fraction were extracted from MCF10A (10A) and MCF10A-CA1 (CA1) cells using Protein and RNA Isolation System (PARIS) Kit (Life technologies Catalog No. AM1921). For whole cell lysate and for each cytoplasmic and nuclear fraction from $10 \mathrm{~A}$ and CA1 cells, $87.5 \%$ of the sample was used for RNA extraction, while $12.5 \%$ was used for an immunoblot to ensure the fractionation was done correctly. The 7:1 ratio was used to maximize RNA content as lncRNAs are relatively lowly expressed. GAPDH (Cell Signaling 14C10) was utilized as control for cytoplasmic fraction, while Tri-Methyl Histone H3 (Lys 27) (Cell Signaling C36B11) was used as control for nuclear fraction for the immunoblot.
RNA was isolated from each fraction using Omega BioTek E.Z.N.A. Total RNA Kit (Catalog No. R6-834-02). Reverse transcription was performed with the Applied Biosystems High-Capacity cDNA Reverse Transcription Kit (Life Technologies Catalog No. 4368813) on the extracted RNA from each compartment as well as the whole cell lysate and RT-qPCR were carried out to analyze the localization of these SE-lncRNAs within the $10 \mathrm{~A}$ and CA1 cells as well as fold change. $18 \mathrm{~s}$, Actin$\mathrm{B}$, HOTAIR were used as controls for localization. $18 \mathrm{~s}$ and Actin-B were also used as controls for fold change to validate the change in expression we saw in our microarray data.

\section{Patient samples extraction}

Archived Formalin-Fixed Paraffin-Embedded (FFPE) patient samples were acquired from Reading Hospital, PA. RNA was extracted from 24 DCIS and 24 IDC samples using the miRNeasy FFPE Kit supplied by QIAGEN (Cat. No. 217504). Reverse transcription was performed using Applied Biosystems High-Capacity cDNA Reverse Transcription Kit (Life Technologies Catalog No. 4368813) on the extracted RNA from each patient sample and RT-qPCR was performed to analyze expression of the 14 target SE-lncRNAs. 18 s, Actin-B and GAPDH were used as controls for fold change in each sample. Unpaired t-test was performed to obtain significance between expression of SE-lncRNAs in DCIS versus IDC patient Samples.

\section{Quantitative reverse transcription PCR primers}

TaqMan assays were purchased from Thermo Fisher Scientific (Waltham, MA), 18S (Hs99999901_s1), Actin (Hs99999903_m1), glyceraldehyde 3-phosphate dehydrogenase (GAPDH; Hs02758991_g1), HOTAIR (Hs03296680), U6 snRNA (Catalog No. 4427975), miR200b (hsa-miR-200b, Catalog No. 4427975). Sybr-green assays purchased from Integrated DNA Technologies (Coralville, IA):

\begin{tabular}{ll}
\hline AC053503.6 & Forward Primer: 5'-AGGTGGATTAGA \\
& GGGGGTGT-3' \\
& Reverse Primer: 5'-GGCTGAGAA \\
& GGGGGTTTCTG-3' \\
& Forward Primer: 5'-CCCGTCGTGACC \\
& TCATTGTG-3' \\
AC068580.7 & Reverse Primer: 5'-GAACCCCTTTTC \\
& CTCACCCA-3' \\
& Forward Primer: 5'-CAAGCTGGA \\
CCND2-AS1 & ACCCTGCAAGA-3' \\
& Reverse Primer: 5'-AAGGGTATACCT \\
& TCCTCCCCA-3' \\
&
\end{tabular}




\begin{tabular}{|c|c|c|c|}
\hline \multirow[t]{2}{*}{ CTD-2033D15.1 } & $\begin{array}{l}\text { Forward Primer: 5'-GGTAAGAAG } \\
\text { CAAAGCCCTGGA3' }\end{array}$ & & $\begin{array}{l}\text { Reverse Primer: 5'-CTGAGCCTGCAA } \\
\text { CTCGACTG-3' }\end{array}$ \\
\hline & $\begin{array}{l}\text { Reverse Primer: 5'-TGGCTGAGACGC } \\
\text { CATCTGTA-3' }\end{array}$ & RP11-323N12.5 & $\begin{array}{l}\text { Forward Primer: 5'-TGGACCAGTCGA } \\
\text { AACCCTTG-3' }\end{array}$ \\
\hline \multirow[t]{2}{*}{ FAM83H-AS1 } & $\begin{array}{l}\text { Forward Primer: 5'-GCAACACCCTAC } \\
\text { TGACCTTGT-3' }\end{array}$ & & $\begin{array}{l}\text { Reverse Primer: 5'-TCTCGACATCGA } \\
\text { GGACCCAT-3' }\end{array}$ \\
\hline & $\begin{array}{l}\text { Reverse Primer: 5'-AGCTCTGTGGTG } \\
\text { ACTGTCTT-3' }\end{array}$ & RP11-326G21.1 & $\begin{array}{l}\text { Forward Primer: 5'-ACTCCGCATTAC } \\
\text { ACCACTGA-3' }\end{array}$ \\
\hline \multirow[t]{2}{*}{ FAM87A } & $\begin{array}{l}\text { Forward Primer: 5'-TTCCGCAGGTTT } \\
\text { TAGTGGCT-3' }\end{array}$ & & $\begin{array}{l}\text { Reverse Primer: } \text { 5'-CCCGAAACAGTA } \\
\text { CCAGGCAA-3' }\end{array}$ \\
\hline & $\begin{array}{l}\text { Reverse Primer: 5'-CAAACTGTCCCC } \\
\text { AACTCCCA-3' }\end{array}$ & RP11-346D6.6 & $\begin{array}{l}\text { Forward Primer: } 5^{\prime}-C A A G C A G C C \\
\text { CTGGAGAGTTTA-3' }\end{array}$ \\
\hline \multirow[t]{2}{*}{ GATA2-AS1 } & $\begin{array}{l}\text { Forward Primer: 5'-GACCCTCTGAAA } \\
\text { GACACCGC-3' }\end{array}$ & & $\begin{array}{l}\text { Reverse Primer: 5'-AACTTGGGGGTC } \\
\text { ACAGCATC-3' }\end{array}$ \\
\hline & $\begin{array}{l}\text { Reverse Primer: 5'-TCTTGCTCATGT } \\
\text { GTGAGGGC-3' }\end{array}$ & RP11-373D23.3 & $\begin{array}{l}\text { Forward Primer: 5'-CTTCCAAGGCCC } \\
\text { TGCATGAT-3' }\end{array}$ \\
\hline \multirow[t]{2}{*}{ HCG9 } & $\begin{array}{l}\text { Forward Primer: 5'-CAGGAACCC } \\
\text { AGGGACTTCAG-3' }\end{array}$ & & $\begin{array}{l}\text { Reverse Primer: 5'-GGTGAGGGA } \\
\text { AGACAACACGG-3' }\end{array}$ \\
\hline & $\begin{array}{l}\text { Reverse Primer: 5'-TGTTCTCTGCAG } \\
\text { CTTGACCT-3' }\end{array}$ & RP11-379F4.4 & $\begin{array}{l}\text { Forward Primer: 5'-TGCCCGGTTTTA } \\
\text { TAGCCCTG-3' }\end{array}$ \\
\hline \multirow[t]{2}{*}{ HOXA11-AS } & $\begin{array}{l}\text { Forward Primer: 5'-TCCGATTTGCAC } \\
\text { GGTGACTT-3' }\end{array}$ & & $\begin{array}{l}\text { Reverse Primer: 5'-ATCTGTTCCGTG } \\
\text { CTCCCTTC-3' }\end{array}$ \\
\hline & $\begin{array}{l}\text { Reverse Primer: 5'-CGGATGTCAGCG } \\
\text { CCTCTAAA-3' }\end{array}$ & RP11-403A21.1 & $\begin{array}{l}\text { Forward Primer: 5'-AGGGATGGG } \\
\text { GTCTCGAGTTT-3' }\end{array}$ \\
\hline \multirow[t]{2}{*}{ LINC00885 } & $\begin{array}{l}\text { Forward Primer: 5'-GGCACTGTAGAA } \\
\text { GCCCCATT-3' }\end{array}$ & & $\begin{array}{l}\text { Reverse Primer: 5'-TCAGCTGGTGGG } \\
\text { TGTTTAGC-3' }\end{array}$ \\
\hline & $\begin{array}{l}\text { Reverse Primer: 5'-GTCCAGCGAACT } \\
\text { GAAGGACA-3' }\end{array}$ & RP11-465B22.8 & $\begin{array}{l}\text { Forward Primer: 5'-AGCCTGAGCTCA } \\
\text { TCCAACAC-3' }\end{array}$ \\
\hline \multirow[t]{2}{*}{ LINC01125 } & $\begin{array}{l}\text { Forward Primer: 5'-AGGCAAAGATGA } \\
\text { GCAGAGCC-3' }\end{array}$ & & $\begin{array}{l}\text { Reverse Primer: 5'-GTGCGTGAACTG } \\
\text { CAGACTTT-3' }\end{array}$ \\
\hline & $\begin{array}{l}\text { Reverse Primer: 5'-CCAAGCAATGCT } \\
\text { GGTTCCTTT-3' }\end{array}$ & RP3-483K16.4 & $\begin{array}{l}\text { Forward Primer: 5-'AGTTGCCATTGA } \\
\text { GCTCCACAA-3' }\end{array}$ \\
\hline \multirow[t]{2}{*}{ LINC01589 } & $\begin{array}{l}\text { Forward Primer: 5'-AAATGGAATGCA } \\
\text { GCCACACC-3' }\end{array}$ & & $\begin{array}{l}\text { Reverse Primer: 5'-TGGACTACTGGC } \\
\text { AGAAGCGT-3' }\end{array}$ \\
\hline & $\begin{array}{l}\text { Reverse Primer: 5'-CCAAGAGGCCAT } \\
\text { CCGTCTTC-3' }\end{array}$ & RP11-507M3.1 & $\begin{array}{l}\text { Forward Primer: 5'-CGCATTTTCCTG } \\
\text { ATTGGCCC-3' }\end{array}$ \\
\hline \multirow[t]{2}{*}{ NR2F1-AS1 } & $\begin{array}{l}\text { Forward Primer: 5'-GGTCACGGA } \\
\text { GAAAACAGGTTCA-3' }\end{array}$ & & $\begin{array}{l}\text { Reverse Primer: 5'-ACATTCCCCTTC } \\
\text { AACGCCTG-3' }\end{array}$ \\
\hline & $\begin{array}{l}\text { Reverse Primer: 5'-CCCCAGAGCTGC } \\
\text { ATCCTTATG-3' }\end{array}$ & RP11-560J1.2 & $\begin{array}{l}\text { Forward Primer: } 5^{\prime} \text {-CCTAGGGTAGTC } \\
\text { CGAGGTCA-3' }\end{array}$ \\
\hline \multirow[t]{2}{*}{ OSMR-AS1 } & $\begin{array}{l}\text { Forward Primer: 5'-TTGGAAACCGAA } \\
\text { AACTCGGC-3' }\end{array}$ & & $\begin{array}{l}\text { Reverse Primer:5'ACAAAATACGCC } \\
\text { CGGCAAAG-3' }\end{array}$ \\
\hline & $\begin{array}{l}\text { Reverse Primer: 5'-ACATTGGGATGT } \\
\text { TCTGCCCC-3' }\end{array}$ & RP11-61F12.1 & $\begin{array}{l}\text { Forward Primer: 5'-GGACGTGGTTTG } \\
\text { CTAGGTGA-3' }\end{array}$ \\
\hline \multirow[t]{2}{*}{ PCAT1 } & $\begin{array}{l}\text { Forward Primer: 5'-CCTCTAAGTGCC } \\
\text { AGTGCAGG-3' }\end{array}$ & & $\begin{array}{l}\text { Reverse Primer: 5'-ACAGGTTTTCCG } \\
\text { TCTCCGAC-3' }\end{array}$ \\
\hline & $\begin{array}{l}\text { Reverse Primer: 5'-ATGTATCTGCGC } \\
\text { ACCCTTTGA-3' }\end{array}$ & RP11-63G10.2 & $\begin{array}{l}\text { Forward Primer: 5'-ACCTGTGCCAGT } \\
\text { GTGAACAA-3' }\end{array}$ \\
\hline \multirow[t]{2}{*}{ RP11-107N15.1 } & $\begin{array}{l}\text { Forward Primer: 5'-GGGTCCTCAATG } \\
\text { TGGGGTTT-3' }\end{array}$ & & $\begin{array}{l}\text { Reverse Primer: 5'-GGGCTAGTCAAA } \\
\text { GTCAGCGT-3' }\end{array}$ \\
\hline & $\begin{array}{l}\text { Reverse Primer: 5'-TCGCTAGAGTCA } \\
\text { CCCCAGTT-3' }\end{array}$ & SLC44A3-AS1 & $\begin{array}{l}\text { Forward Primer: 5'-AGCAACAGTGTA } \\
\text { GTGGCGTA-3' }\end{array}$ \\
\hline \multirow[t]{2}{*}{ RP11-258F1.1 } & $\begin{array}{l}\text { Forward Primer: 5'-CGTTGTACAGGC } \\
\text { CCTTCTCA-3' }\end{array}$ & & $\begin{array}{l}\text { Reverse Primer: 5'-CTGGCCTGTGAT } \\
\text { GCTTTTCC-3' }\end{array}$ \\
\hline & $\begin{array}{l}\text { Reverse Primer: 5'-GTGCGCACAACC } \\
\text { CTGGTATC-3' }\end{array}$ & SNHG18 & $\begin{array}{l}\text { Forward Primer: 5'-CATGTTCCCAGA } \\
\text { GGTTGGCA-3' }\end{array}$ \\
\hline RP11-303E16.3 & $\begin{array}{l}\text { Forward Primer: 5'-CAGACTCCGTAC } \\
\text { GCCTTCAC-3' }\end{array}$ & & $\begin{array}{l}\text { Reverse Primer: 5'-AGAGGACAA } \\
\text { GGCAAAACACTT-3' }\end{array}$ \\
\hline
\end{tabular}




\begin{tabular}{ll}
\hline TMEM220-AS1 & Forward Primer: 5'-TCCAAGTCCCCT \\
& TCTGACTTC-3' \\
& Reverse Primer: 5'-CAGGCTCCTCAG \\
& GAAGAATCC-3' \\
& Forward Primer: 5'-GGCAGTGTAGCG \\
& AGAAAGT-3' \\
SNORD3B-2 & Reverse Primer: 5'-AATAGGAGGTGC \\
& CACACAGC-3' \\
& Forward Primer: 5'-CGCTACAACCCA \\
RARRES1 & GAGTCTTAC-3' \\
& Reverse Primes: 5'-TCACACTAGTGA \\
& GCTGTGCC-3' \\
\hline
\end{tabular}

\section{Knockdown of potential cis-acting SE-IncRNAs}

150,000 DCIS and CA1 cells were plated into a six well plate using MFC10A media and transfected with 10 $\mathrm{uL}$ of $10 \mathrm{uM}$ Gapmers or Antisense Oligonucleotides (ASOs) using $7.5 \mathrm{uL}$ of RNAiMax Lipofectamine (Invitrogen Catalog No. 13778150). Cells were collected 48 h post-transfection and RNA was isolated using miRNeasy Mini Kit (Qiagen Catalog No. 217004). Reverse transcription of the collected RNA was performed using Applied Biosystems High-Capacity cDNA Reverse Transcription Kit (Life Technologies Catalog No. 4368813). RT-qPCR was performed using the primers mentioned above to analyze expression of the SE-lncRNAs and their neighboring mRNA after knockdown. U6 small nucleolar RNA was used as control for miR-200b expression while $18 \mathrm{~s}$, Actin-B and GAPDH were used as controls to assess knockdown of the SE-lncRNAs and expression of RARRES1 mRNA.

SE-IncRNA LNA Gapmers:

\begin{tabular}{ll}
\hline RP11-379F4.4: & Gapmer 1: 5'-ACTAGGTCCGAGGCAA-3' (Qiagen Catalog \\
& No. 339511 LG00247071) \\
& Gapmer 2: 5'-ATGACTAAGGAACTAG-3' (Qiagen Catalog \\
& No. 339511 LG00247084) \\
RP11-465B22.8: & Gapmer 1: 5'-GCGGTGAGGAGGTGCT-3' (Qiagen Catalog \\
& No. 339511 LG00247067) \\
& Gapmer 2: 5'-GTGCGTGAACTGCAGA-3' (Qiagen Catalog \\
& No. 339511 LG00247383) \\
\hline
\end{tabular}

\section{Chromatin immunoprecipitation (ChIP)}

MCF10A, MCF10A-AT1, DCIS.com, and MCF10A-CA1 cells were grown to a final count of $5 \times 10^{6}$. Cells were chemically crosslinked by the addition of $1 \mathrm{~mL}$ of fresh $10 \%$ formaldehyde solution for $10 \mathrm{~min}$ at room temperature on a rocker. After $10 \mathrm{~min} 1 / 10$ volume of $1.25 \mathrm{M}$ Glycine was added to quench unreacted formaldehyde and incubated for $5 \mathrm{~min}$ on rocker. Cells were pelleted at $1000 \mathrm{~g}$ for $5 \mathrm{~min}$, washed twice with $1 \times \mathrm{PBS}$, flash frozen in liquid nitrogen, and stored at $-80{ }^{\circ} \mathrm{C}$ prior to use. Cells were resuspended, lysed in lysis buffer $(50 \mathrm{mM}$ HEPES, $140 \mathrm{mM} \mathrm{NaCl}, 1 \mathrm{mM}$ EDTA, 1\% Triton X-100, $0.1 \%$ Sodium deoxycholate, $0.1 \%$ SDS), and sonicated to solubilize and shear crosslinked DNA. Sonication conditions vary depending on cells, but cells were sonicated using a Diagenode Bioruptor Sonicator and sonicated at power 7 for $13 \times 30 \mathrm{~s}$ pulses ( $30 \mathrm{~s}$ pause between pulses) at $4{ }^{\circ} \mathrm{C}$ while samples were immersed in an ice bath. The sonicated cells were centrifuged for $10 \mathrm{~min}$ at $8000 \mathrm{~g}$ at $4{ }^{\circ} \mathrm{C}$ and the supernatant collected to proceed with immunoprecipitation. The resulting whole-cell extract volume was divided into two, one for IgG and the other for H3K27ac targeting. The samples were diluted in 1:10 ratio with RIPA buffer $(50 \mathrm{mM}$ Tris- $\mathrm{HCl} \mathrm{pH} 8,150 \mathrm{mM}$ $\mathrm{NaCl}, 2$ mM EDTA pH8, 1\% NP-40, 0.5\% Sodium Deoxycholate, $0.1 \%$ SDS) incubated overnight at $4{ }^{\circ} \mathrm{C}$ with the $2.5 \mu \mathrm{g}$ of the appropriate antibody, Cell Signaling IgG (Rabbit (DA1E) mAb IgG XP ${ }^{\circledR}$ Isotype Control \#3900) and Abcam H3K27ac (Anti-Histone H3 (acetyl K27) antibody-ChIP Grade (ab4729)). The following day, $60 \mu \mathrm{L}$ of ChIP Grade Protein G Magnetic beads (Cell Signaling 9006S) were washed three times with RIPA buffer and $30 \mu \mathrm{L}$ each of the washed beads were added to IgG and $\mathrm{H} 3 \mathrm{~K} 27 \mathrm{ac}$ samples and left rotating at $4{ }^{\circ} \mathrm{C}$ for $3 \mathrm{~h}$. IgG and H3K27ac samples with magnetic beads were then washed three times with low salt wash buffer $(0.1 \%$ SDS, 1\% Triton X-100, 2 mM EDTA, 20 mM Tris-HCl pH 8.0, $150 \mathrm{mM} \mathrm{NaCl}$ ) and one time with high salt wash buffer (0.1\% SDS, 1\% Triton X-100, 2 mM EDTA, 20 mM Tris$\mathrm{HCl} \mathrm{pH} \mathrm{8.0,500} \mathrm{mM} \mathrm{NaCl).} \mathrm{DNA} \mathrm{was} \mathrm{then} \mathrm{eluted} \mathrm{off} \mathrm{the}$ beads for each sample by heating at $65^{\circ} \mathrm{C}$ at $1200 \mathrm{~g}$ for $1 \mathrm{~h}$, cooling each sample at room temp for $2 \mathrm{~min}$, centrifuging for $1 \mathrm{~min}$ at $10,000 \mathrm{~g}$, and putting each sample on a magnet for $2 \mathrm{~min}$ and removing the liquid. $4.8 \mu \mathrm{L}$ of $5 \mathrm{M} \mathrm{NaCl}$ and $2 \mu \mathrm{L}$ RNase A $(10 \mathrm{mg} / \mathrm{mL})$ was added to each sample and incubated while shaking at $1200 \mathrm{rpm}$, $65{ }^{\circ} \mathrm{C}$ overnight. The next day, $2 \mu \mathrm{L}$ proteinase $\mathrm{K}(20 \mathrm{mg} /$ $\mathrm{mL}$ ) was added to each sample and incubated while shaking at $1400 \mathrm{rpm}, 60^{\circ} \mathrm{C}$ for $1 \mathrm{~h}$. DNA was purified using a QIAGEN QIAquick PCR purification kit (cat. Number 28104).

\section{ChIP-sequencing sample preparation and analysis}

1 to $10 \mathrm{ng}$ of DNA was prepared for sequencing using NEBNext Ultra II DNA Library Prep Kit (E7645S) and NEBNext ${ }^{\circledR}$ Multiplex Oligos for Illumina ${ }^{\circledR}$ (Index Primers Set 1) (E7335S). DNA was sequenced at the UAlbany Center for Functional Genomics using the Illumina NextSeq 500 with single end 75 bp reads. Quality of samples for ChIP was assessed using the Bioconductor package FastQC (version 0.11.9). Data were mapped to the human reference genome (hg38 assembly) using STAR Aligner (version 2.7.0). PCR duplicated reads were filtered using 
Sambamba (version 0.7.1). The MACS2 (version 2.2.7.1) algorithm was used to identify enriched regions (peaks). Default parameters were used with q-value of 0.05 except to ensure broad regions were identified; broad peak calling was added with a broad cutoff of 0.05 . Reads were normalized to mapped reads.

\section{Super-enhancer identification}

Super-enhancers were defined by stitching peaks using Rank Order Super-Enhancer (ROSE) (version python 2.7.3) with default parameters, except TSS exclusion zone size was adjusted to $250 \mathrm{bp}$. TSS exclusion was used because the H3K27ac signal is enriched for both active enhancers and promoters. However, several highthroughput reporter studies in mammals assessing either selected genomic regions (e.g., open chromatin regions or transcription factor binding sites) or human whole genomes have also found a substantial proportion of enhancers overlapping TSS-proximal regions $[18,19]$. Signals for super-enhancers identified in each cell line were quantified in progression. Briefly, super-enhancer bed files for each cell line were collapsed into AllSEs.bed using bedtools merge. Coverage of AllSEs.bed was quantified using bam files for each cell line in the progression series using bedtools intersect. Reads were normalized to the sequencing depth, and fold-change was calculated. H3K27ac ChIP seq data for ER + patients was obtained from European Nucleotide Archive under project no. PRJEB22757 and for TNBC patient samples and TNBC cell lines under project no. PRJEB33558.

\section{Acquired/lost super-enhancer identification}

Acquired super-enhancer regions were classified at the AT1 stage in progression by comparing super-enhancers ranked in AT1 cells with super-enhancers ranked in MCF10A cells and only keeping those that were ranked as super-enhancers in AT1 but not in MCF10A cells using bedtools intersect. Similarly, super-enhancers acquired at the DCIS stage were those that were ranked in DCIS cells but were not ranked in MCF10A and AT1 cells. Lastly, super-enhancers acquired at the CA1 stage were only ranked in CA1 cells but not in MCF10A, AT1, and DCIS cells. Lost super-enhancers were identified by comparing super-enhancers ranked in AT1, DCIS, and CA1 cells with super-enhancers in MCF10A cells and observing those that were not present in the corresponding cells but were in the normal MCF10A cells. Genes within $50 \mathrm{~kb}$ of the acquired/lost super-enhancers were classified using bedtools closest function. Gene Ontology (GO) analysis of these genes was performed using ENRICHR.

\section{Statistical analysis}

All experiments were run in triplicate, except ChIP seq, which were done in duplicates. Data are represented as the mean \pm standard deviation (mean $\pm \mathrm{SD}$ ). All statistical analyses were carried out using GraphPad Prism 9 Software (La Jolla, CA, USA). Statistical significance for SE-lncRNAs and mRNAs expression in our progression series were characterized by one-way ANOVA with Tukey correction. All significant and non-significant results are shown in Additional file: 7 Table S4. Expression of 14 target SE-lncRNAs in FFPE DCIS and IDC patient samples was analyzed by unpaired Student's $t$-test and $p$-values are listed below in Fig. $4 \mathrm{~d}$ and e for our two promising targets, while rest are shown in Additional file 2: Figure S2. Paired Student's $t$-test was used to analyze knockdown of the two promising SE-lncRNAs as well as the corresponding expression of their associated mRNA, $p<0.05$ (compared with the control antisense oligonucleotide) was considered significant and is marked with an asterisk in the figures.

\section{Results}

Global analysis of SE-IncRNAs acquired in IDC progression Underlying mechanisms that support breast cancer progression have been well studied. However, clear functional determinants segregating non-invasive from invasive tumors have yet to be defined. Acquired lncRNAs transcribed from super-enhancer loci can lead to discovering markers of progression, improving breast cancer diagnostics and treatment for patients. The MCF10A progression series mimics progression of breast cancer originating within the epithelial cells of the mammary ducts. The progression series was originally generated from MCF10A (10A) cells, a spontaneously immortalized mammary epithelial cell line derived from benign breast tissue from a woman with fibrocystic disease [20]. MCF10A cells were transformed with oncogenic HRAS to generate MCF10AT1 pre-malignant cells that form atypical ductal hyperplasia in mice [20]. MCF10DCIS.com (DCIS or DCIS.com) cells were derived from MCF10AT1 xenograft model and form predominantly comedo DCIS when injected into mice [21]. MCF10CA1 (CA1) cells, derived from MCF10AT1, form poorly differentiated malignant tumors in xenograft models [22]. Arraystar has developed a platform to comprehensively study super-enhancer lncRNAs and their downstream targets [23]. Taking advantage of this commercially available approach, we assessed SE-lncRNA expression within the MCF10A progression series. This analysis interrogated 7753 SE-lncRNAs (Fig. 1a), as well as 7040 associated mRNAs [24].

We found that super-enhancer associated lncRNAs are dynamically expressed during breast cancer progression. 

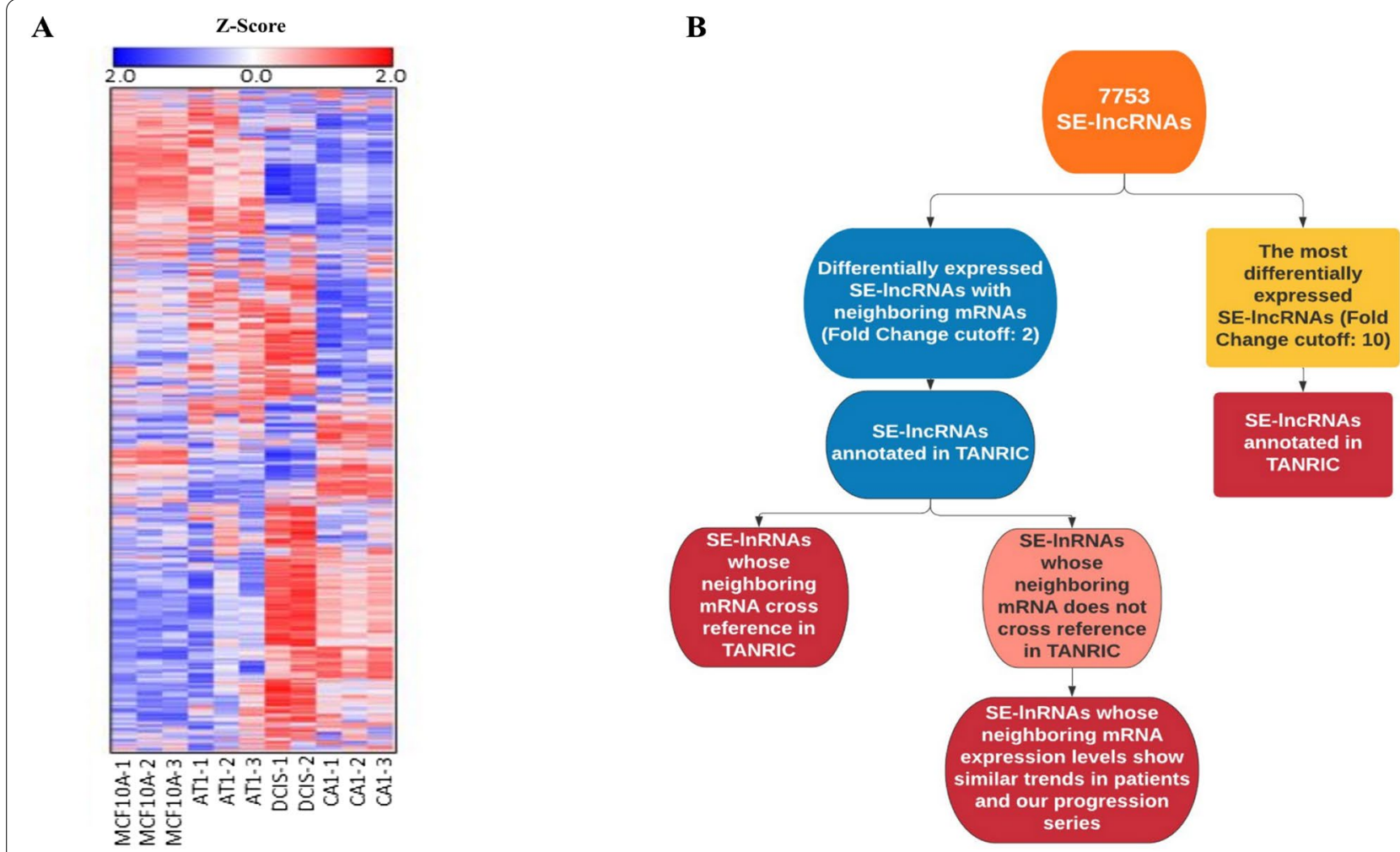

C

FZD7 JARID2 USP22 KCTD5 BDH1 SOGA2 NR2F1 PXDC1 ETNK2 ZNF816 ELOVL5 THBS1 BMP2K TNFAIP2 KIAA0196 C4B MFSD1 AHR SULF2 KIAA0196 CCND2 HOXA11 CALML3 SH3GLB1 ADRM1 TIMP2 SLC9A9 ITM2C NBPF16 ZNF706 KLF7 PMEPA1 FBX032 LIPA MYH3 FAM228B PMEPA1 FNBP1 TNS4 LAMA3 VSIG8 HOXC11 HOXA11 ZNFX1 SAP30 RHOB FAM84B BAHD1 BCL10 GNA15 FAM13A CENPN RAI14 CRIM1 IRS1 FAM213A OSMR TNFRSF10B ARSG CTDSP1 IGFL1 DKK1 RAB3A ZNFX1 XRCC6BP1 SEMA5A PHLDA1 TMEM63A UBAC2 SIX2 SLC44A3 ZCCHC7 ABLIM3 ACTR1B SLC44A3 CAPN10 ANK2 STEAP1B ZMYND8 IL4R UBE2V1 MARCO NFAM1 IER5 IRF2BPL EXTL2 LIF EGFR DES MPHOSPH6 CTSD HLA-A POFUT2 BTN1A1 VPS37B SLC22A5 LIF NCOA3 S100A4 SLC44A3 PLXNC1 EPCAM WDR81 MYLK NDFIP2 RIMBP2 PDE4DIP DUSP5 EDN1 LRRFIP2 HLA-A DUSP5 LAPTM4A DSP LGALS8 GFOD1 KBTBD11 OGFRL1

\begin{tabular}{clrrr} 
Index & Name & P-value & $\begin{array}{c}\text { Odds } \\
\text { Ratio }\end{array}$ & $\begin{array}{c}\text { Combined } \\
\text { score }\end{array}$ \\
\hline 1 & Synthesis and degradation of ketone & 0.06126 & 15.87 & 44.33 \\
2 & bodies & 0.01062 & 6.61 & 30.06 \\
3 & p53 signaling pathway & 0.02751 & 7.74 & 27.82 \\
4 & JaK-STAT signaling pathway & 0.003645 & 4.90 & 27.51 \\
5 & Lysosome & 0.007662 & 5.16 & 25.15 \\
6 & FoxO signaling pathway & 0.009768 & 4.81 & 22.26 \\
7 & MicroRNAs in cancer & 0.002892 & 3.72 & 21.72 \\
8 & Estrogen signaling pathway & 0.01108 & 4.63 & 20.87 \\
9 & Focal adhesion & 0.008587 & 3.99 & 18.97 \\
10 & Steroid biosynthesis & 0.1132 & 8.35 & 18.20
\end{tabular}

Fig. 1 Filtering process taken to identify potentially cis-acting SE-IncRNAs from 7753 SE-IncRNAs that may contribute to progression to early-stage breast cancer. a Heatmap of 7753 SE-IncRNAs in MCF10A progression series. Hierarchical Clustering was performed. b Process to identify potentially cis-acting SE-IncRNAs crucial for progression of DCIS to IDC. Red boxes represent SE-IncRNAs that made the "cut." c Gene Ontology analysis on the list of 138 mRNAs that were neighboring differentially expressed SE-IncRNAs (Fold Change cutoff: 2) 
There are patterns of both SE-lncRNAs being acquired and lost as well as several SE-lncRNAs that exhibit a stepwise gain or loss in expression in progression (Additional file 4: Table S1). While the heat map from our analysis (Fig. 1a) demonstrates dynamic expression changes, the majority of these lncRNAs are not functionally defined.

Enhancer elements may become engaged either distally or locally to impact target gene expression. Transacting lncRNAs are transcribed, processed, and then vacate their sites of transcription to exert their function elsewhere, akin to mRNAs. Their final destination, be it in the cytoplasm or nucleus, does not depend on their transcription site [25]. By contrast, cis-acting lncRNAs are those whose activity is based at and dependent on the loci from which they are transcribed. Transcripts with the potential of acting in cis likely make up a substantial portion of known lncRNAs: the majority of lncRNAs are enriched in the chromatin fraction, and specifically are tethered to chromatin, presumably at their sites of transcription, through Pol II $[25,26]$. If functional, this would indicate that the effects of these lncRNAs are centered at these loci. In addition, the fairly low levels at which lncRNAs are generally expressed, oftentimes just a few molecules per cell, naturally favor a cis mechanism of action, as diffusion or transport to other cellular compartments would render these transcripts too diluted to mediate a plausible function [27]. Identifying distally engaged enhancer elements is difficult since within the threedimensional structure of the nucleus there are a myriad of possible locations they can interact.

To classify super-enhancers that may become locally engaged and regulate cancer progression, we applied filters to identify potential cis-acting SE-lncRNAs (Fig. 1b). First, from the 7753 SE-lncRNAs screened (Fig. 1b), we sorted and extracted SE-lncRNAs that are up or down regulated from normal 10A cells to invasive CA1 cells (fold change cut-off: \pm 2 ). Furthermore, from these SElncRNAs that met our cutoff, we identified those which have neighboring genes within 50 kilo-bases upstream or downstream of the lncRNA that also demonstrate changing expression. The $50 \mathrm{~kb}$ window upstream and downstream were assigned as enhancers tend to loop to and associate with adjacent genes in order to activate their transcription [28] and primarily these interactions occur within a distance of $\sim 50 \mathrm{~kb}$ of the enhancer locus [29]. This allowed us to identify 138 SE-lncRNAs (Additional file 5: Table S2). Gene Ontology (GO) assessment on the list of mRNAs was performed to provide insight into what pathways the SE-lncRNAs might be regulating (Fig. 1c). Many pathways that appear in our GO analysis, such as focal adhesion, p53 signaling pathway, and JAK-STAT signaling pathway, constitute a major group of related signaling pathways that control proliferation, survival, angiogenesis, and metastasis of breast cancer, suggesting these SE-lncRNAs could be regulating canonical cancer promoting genes.

\section{Identifying clinically relevant potentially cis-acting SE-IncRNAs}

To give clinical relevance to our data, these 138 SE-lncRNAs were cross-referenced with The Atlas of Non-coding RNAs In Cancer (TANRIC), which compiles patient data from The Cancer Genome Atlas (TCGA), and data from Cancer Cell Line Encyclopedia (CCLE) [30]. From our list of 138 SE-lncRNAs that are associated with mRNAs within the MCF10A series, we identified 27 SE-lncRNAs that are annotated in patient samples within TANRIC (Fig. 2) (Additional file 6: Table S3). If a SE-lncRNA is cis-acting and impacting enhancer activity or associated mRNA expression, then a change in SE-lncRNA expression should be accompanied by a change in target mRNA expression. Therefore, we next identified mRNAs whose expression levels correlated in patient samples within all subtypes of breast cancer for each of these 27 SE-lncRNAs (Pearson correlation coefficient $\geq 0.5$ or $\leq-0.5$ ) (Additional file 6: Table S3). This analysis identified 11 SE-lncRNAs as potentially cis-acting (Fig. 3a). The remaining 16 SE-lncRNAs were further filtered out to focus on those whose neighboring mRNAs expression showed similar trends from normal to tumorous cells in patient samples and our progression series (Fig. 3b) (Additional file 6: Table S3). Lastly, we took the most differentiated SE-lncRNAs (Fig. 1b) within the progression series from our array (fold change cut-off: \pm 10 ) and highlighted 4 that were annotated by TANRIC (Fig. 3c). One-way ANOVA with Tukey correction was performed to gauge statistical significance in expression changes in progression for all SE-lncRNAs presented in Fig. 3 and are reported in Additional file 7: Table S4. From our approach to identify potentially cis-acting SE-lncRNAs, we narrowed our list from 7753 to 31, with 27 promising targets as well as 4 SE-lncRNAs selected for follow up due to high differential expression within disease progression.

\section{Characterization of potential cis-acting SE-IncRNAs}

Our approach to identify cis-acting SE-lncRNAs in progression provided 27 potential targets, 11 of which are highly promising, as well as 4 SE-lncRNAs with the highest differential expression within disease progression. Cis-acting SE-lncRNAs are expected to be localized within the nucleus; thus, to focus and narrow our list of potentially cis-acting SE-lncRNAs that are active in progression of DCIS to IDC, we investigated their sub cellular location. Whole cell lysate, as well as separated cytoplasmic fraction and nuclear fraction were extracted from the $10 \mathrm{~A}$ and CA1 cells. These cell lines were chosen 
RP11-107N15.1 RP11-674N23.1 CTD-2033D15.1 RP11-379F4.4 NKILA FAM83H-AS1 RP11-61F12.1 Z83851.1 HOXA11-AS CATG00000062072.1 AK056098 AK056098 HOTAIR RP11-103H7.5 RP11-303E16.3 CTD-2127H9.1 RP11-346D6.6 RP11-290L1.3 LINC01057-1 LINC01057-2 AC002480.3 RP11-77A13.1 RP4-549L203 AC068580.7 RP11-4630123 LINC01057 MYLK-AS1 RP11-525A16.4 RP11-525A16.4 GFOD1-AS1 AC093702.1 RP11-560J1.2 NR2F1-AS1 CATG00000069061.1 CATG00000095263.1 RP1-56K13.2 SNORD3B-2 SRGAP3-AS3 RP11-473M20.7 CALML3-AS1 AC012507.3 RP11-174I12.2 RP11-409K20.6 RP1-170019.14 RP11-64K12.9 CTD-2024P10.1 RP11-875011.3 AC068499.10 RP11-285F7.2 RP11-22011.1 LINC00472 CATG00000053405.1 CTA-126B4.7 RP1-102K2.8 HCG9-1 AC034220.3 RP11-74K11.2 LINC01038 CATG00000082768.1 RP11-644K8.1 KBTBD11-OT1 RP11-344E13.3 RP11-420L9.5 LINC00677 CATG00000053438.1 CATG00000021837.1 RP11-94A24.1 RP11-798M19.6 CTB-43E15.4 RP4-612B15.3 RP11-14N7.2 RP11-149123.3 RP5-1028K7.2 ZFAS1-1 RP11-131L23.1 RP11-490M8.1 WIPI1 ZFAS1-2 UBAC2 CATG00000078011.1 CAPN10-AS1 CATG00000027002.1 RP11-309G3.3 EGFR-AS1 LINC00205 CATG00000058427.1 BCYRN1 RP11-662M24.2 RP11-285J16.1 RP3-512B11.3 CTD-2331H12.7 CTD-3126B10.4 RP11-74C13.4 NSMCE2-1 NSMCE2-2 XLOC_008559 CATG00000048772.1 RP11-437J19.1 RP11-258F1.1 RP11-157P1.4 KB-1460A1.5 CTC-29TN7.7 RP11-403A21.1 RP11-798M19.6 AC005264.2 CATG00000051748.1 RP11-378A13.2 RP11-620J15.3 CATG00000028064.1 LINC01125 CATG00000069800.1 TRERNA1 CATG00000021639.1 AC053503.6 HCG11 LOC101928034 MIR22HG RP11-326G21.1 HCG9-2 RP11-385F5.5 CATG00000053419.1 RP13-61613.1 RP3-483K16.4 CAB-AS1 CCND2-AS1 DLGAP1-AS2 RP11-458N5.1 RP11-256123.3 LINC01730 RP11-323N12.5 AC007879.6 RP11-507M3.1 RP11-190A12.8 CATG00000042058.1 FAM13A-AS1 RP11-137H2.6 AC006262.5 SNHG18

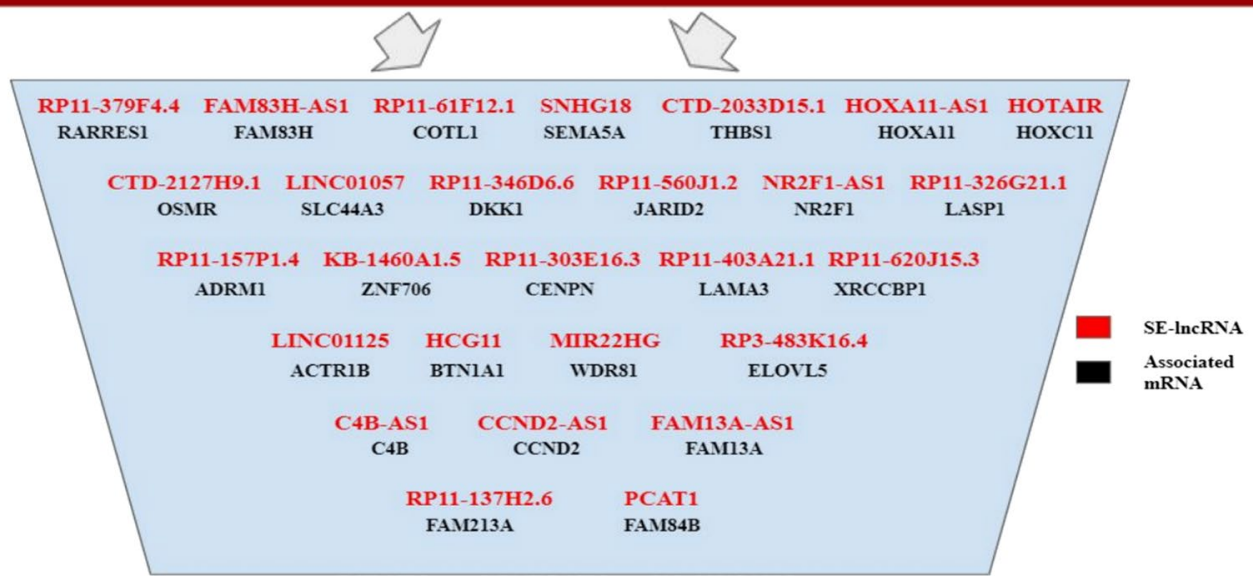

Fig. 2138 SE-IncRNAs filtered to 27 SE-IncRNAs and their neighboring mRNAs. From 138 SE-IncRNAs, 27 potentially cis-acting SE-IncRNAs and their neighboring mRNA were highlighted

to determine if localization changed during progression. For all samples, $87.5 \%$ of the sample was used for RNA extraction, while $12.5 \%$ was used for an immunoblot to ensure the fractionation was done correctly (Fig. 4a). The 7:1 ratio was used to maximize RNA content as lncRNAs are relatively lowly expressed. From the 31 SE-lncRNAs, 14 were primarily localized within the nucleus (Fig. 4b, c). The remaining 17 were either localized within the cytoplasm or were expressed at low levels that localization could not be determined (Additional file 1: Figure S1).

Next, we utilized 24 DCIS and 24 IDC FormalinFixed Paraffin-Embedded (FFPE) patient samples of varying subtypes to assess the expression levels of the 14 SE-lncRNAs. Of the 14 SE-lncRNAs we tested in patient samples, RP11-379F4.4 and RP11-465B22.8 stood out as their increase in expression from DCIS to IDC was statistically significant and the expression levels matched our MCF10A model (Fig. 4d, e). The remaining targets either did not show significant results (Additional file 2: Figure S2) or expression was not determined. Albeit RP11-465B22.8 was not identified with an associated mRNA, the fact that it was the most up-regulated in progression prompted us to scan 50 kilobases upstream and downstream of the gene for potential target genes it might regulate. We discovered that this SE-lncRNA neighbors the miR-200 family of genes of which miR-200b is well studied to have a role as a tumor-suppressor [31] and, in other cases, a tumor promoter [32]. Having narrowed our list to one potential cis-acting SE-lncRNA and one SE-lncRNA that was highest differentiated in our model, we performed knockdown of the two targets and observed the expression of their associated gene in DCIS and CA1 cells (Fig. 4f). A two-fold increase was seen in the expression of RARRES1 $48 \mathrm{~h}$ after knockdown of RP11-379F4.4 in DCIS and CA1 cells. Conversely, about a two-fold decrease in expression was observed for miR-200b $48 \mathrm{~h}$ post knockdown of RP11-465B22.8. These results illustrate that RP11-379F4.4 and RP11-465B22.8 are involved in regulating the expression of their neighboring gene. Importantly, this result shows that the lncRNA transcripts themselves are involved in regulating their neighboring gene and not the act of transcription of the SE-lncRNA itself. We have discovered several SE-lncRNAs that exhibit dynamic expression in 


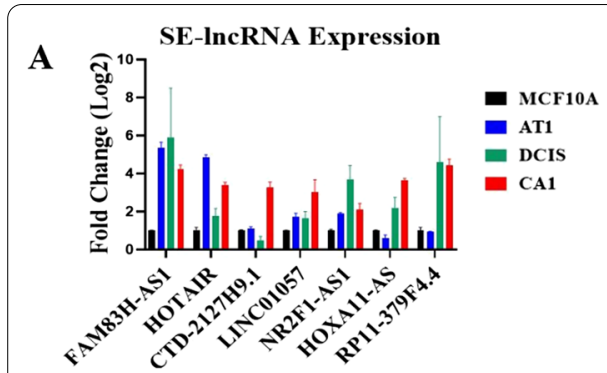

Neighboring mRNA Expression
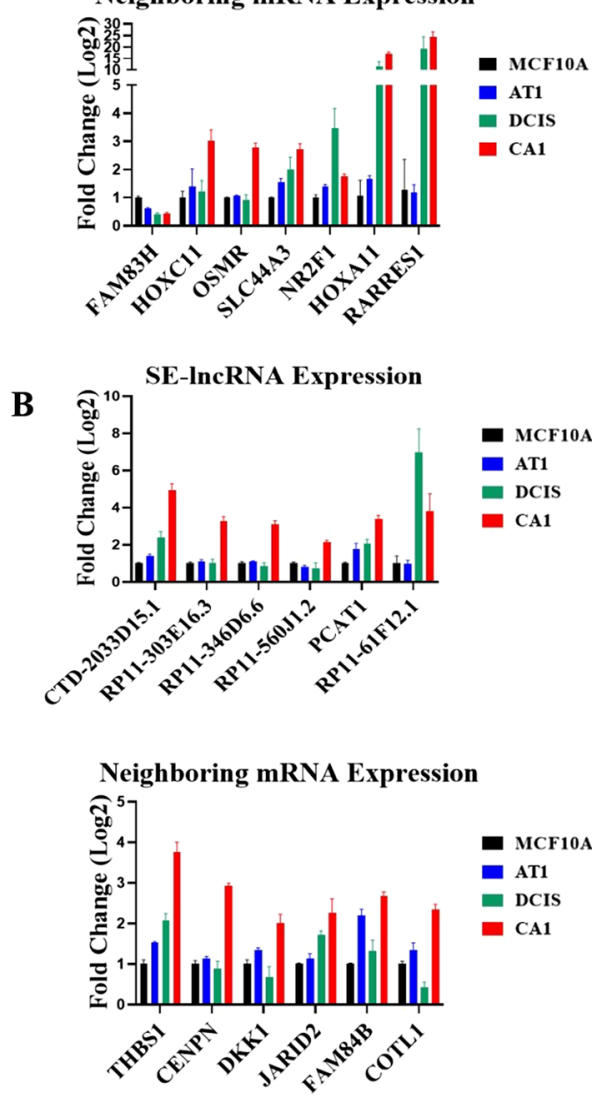
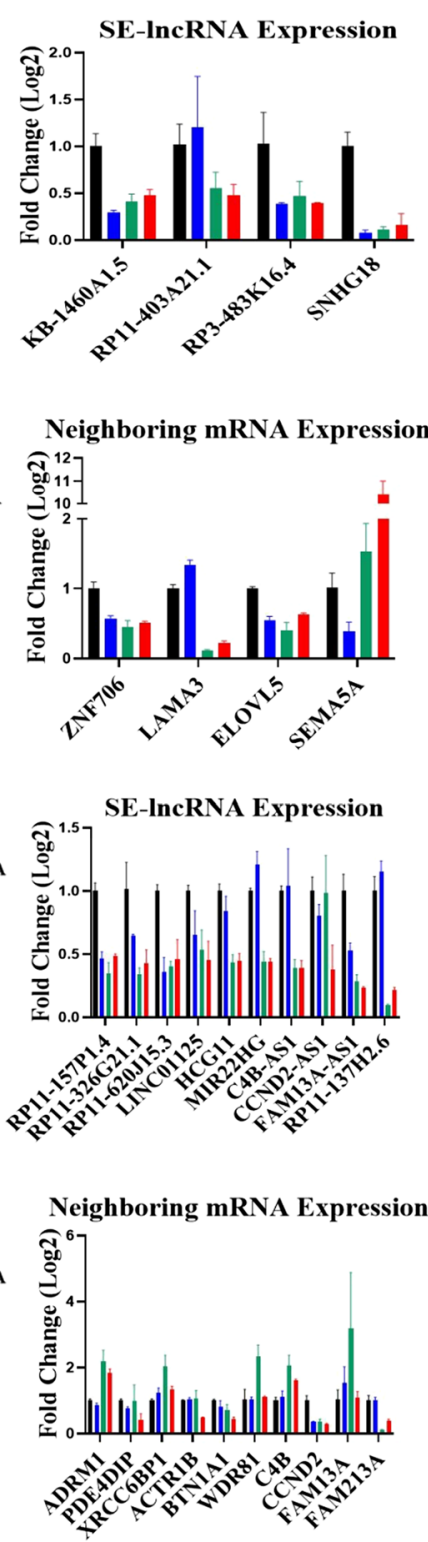

Fig. 3 Expression of 27 potentially cis-acting SE-IncRNAs and their neighboring mRNA and 4 of the highest differentially expressed SE-IncRNAs in the MCF10A progression series. a Expression of 11 SE-IncRNAs and their neighboring mRNAs in progression that correlated in TANRIC. Top are upregulated SE-IncRNAs and their associated mRNA while bottom are down regulated and their associated mRNA. SE-IncRNA-mRNA pairs: FAM83H-AS1 and FAM83H, HOTAIR and HOXC11, CTD-2127H9.1 and OSMR, LINC01057 and SLC44A3, NR2F1-AS1 and NR2F1, HOXA11-AS and HOXA11, RP11-379F4.4 and RARRES1, KB-14601.5 and ZNF706, RP11-403A21.1 and LAMA3, RP3-483K16.4 and ELOVL5, SNHG18 and SEMA5A. b Expression of $16 \mathrm{SE}-\mathrm{InCRNAs}$ and their neighboring mRNAs in progression that did not correlate in TANRIC. Top are upregulated SE-IncRNAs and their associated mRNA, while bottom are down regulated and their associated mRNA. SE-InCRNA-mRNA pairs: CTD-2033D15.1 and THBS1, RP11-303E16.3 and CENPN, RP11-346D6.6 and DKK1, RP11-560J1.2 and JARID2, PCAT1 and FAM84B, RP11-61F12.1 and COTL1, RP11-57P1.4 and ADRM1, RP11-326G21.1 and PDE4DIP, RP11-620J15.3 and XRCC6BP1, LINC01125 and ACTR1B, HCG11 and BTN1A1, MIR22HG and WDR81, C4B-AS1 and C4B, CCND2-AS1 and CCND2, FAM13A-AS1 and FAM13A, RP11-137H2.6 and FAM213A. c Expression of the highest differentiated SE-IncRNAs in progression. Top two are upregulated SE-IncRNAs, while bottom two are down regulated. One-way ANOVA with Tukey correction was carried out to evaluate statistical significance of gene expression between cell lines, $n=3,{ }^{*}=P<0.05$, error bars represent standard deviation. Full statistical analysis is presented in Additional file 6: Table S3 

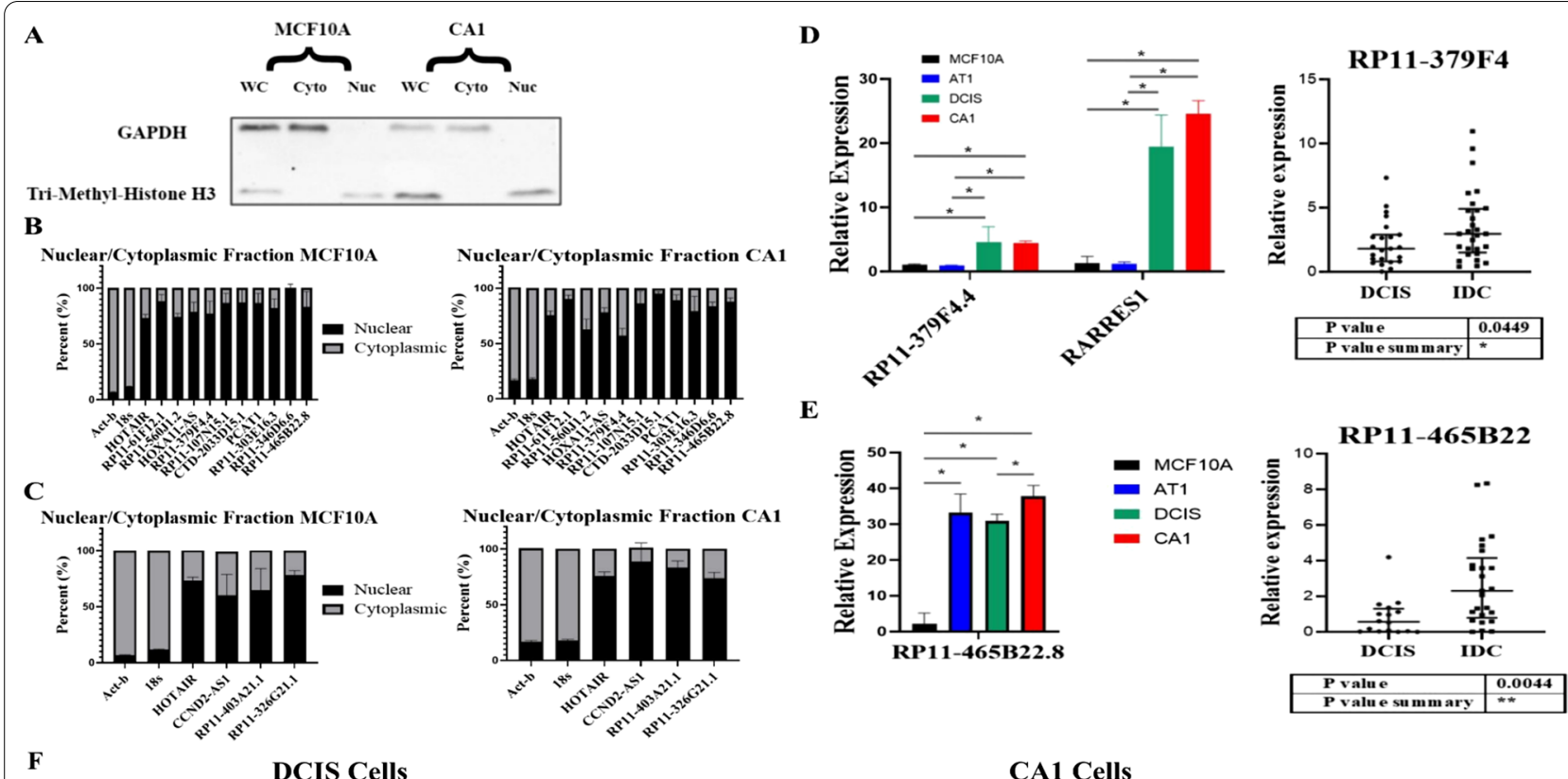

$\mathbf{F}$

\section{DCIS Cells}

CA1 Cells
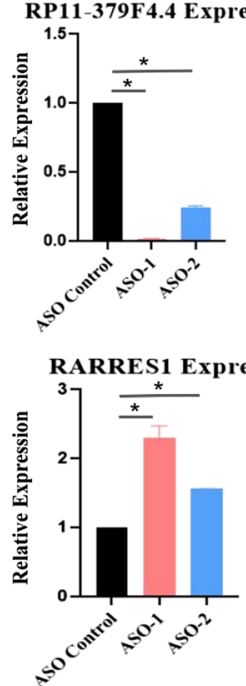
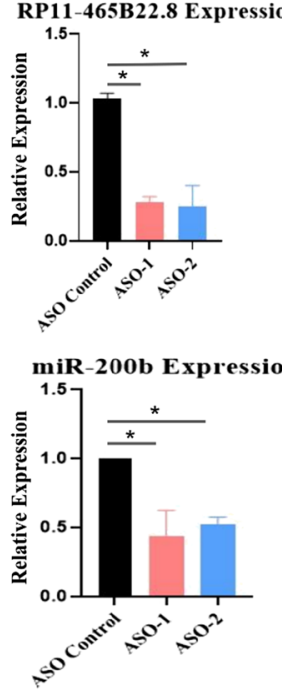
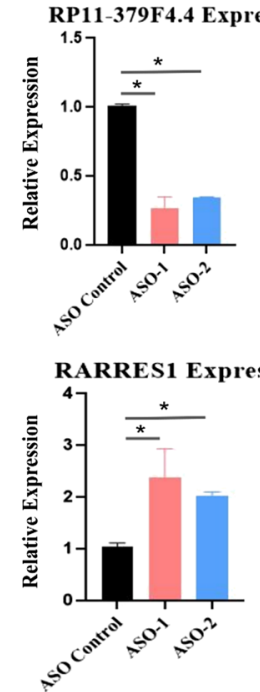
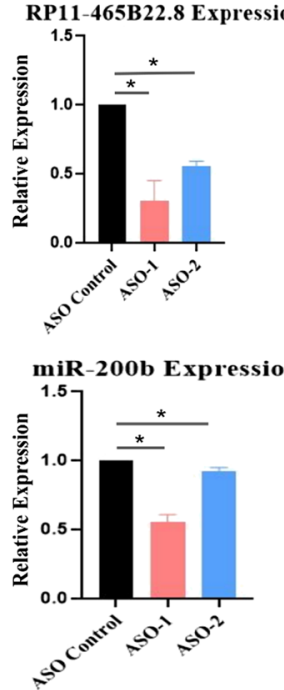

Fig. 4 Localization of the potential cis-acting SE-IncRNAs. a Immunoblot of Cell Fractionation of Whole Cell Lysate, Cytoplasmic Fraction, and Nuclear Fraction in MCF10A and CA1 cells. GAPDH was used as control for Cytoplasmic fraction, while Tri-methyl Histone was used as control for Nuclear Fraction. b, c Localization of 14 SE-IncRNAs (11 up-regulated and 3 down-regulated) from our list of 27 potentially cis-acting SE-IncRNAs and 4 highest differentiated that are primarily localized within the nucleus. $\mathbf{d}$ Expression level of SE-IncRNA, RP11-379F4.1, and its neighboring mRNA, RARRES1, in MFC10A progression series, $n=3,{ }^{*}=P<0.05$, one-way ANOVA with Tukey comparison, error bars represent standard deviation. Expression levels of SE-IncRNA RP1 1-379F4.1 in 24 DCIS and 24 IDC patients ( ${ }^{*}=P<0.05$ ), unpaired t test. e Expression level of the highest differentiated SE-IncRNA, RP1 1-465B22.8, in MCF10A progression series, $n=3,{ }^{*}=P<0.05$, one-way ANOVA with Tukey comparison, error bars represent standard deviation. Expression levels of SE-InCRNA RP11-465B22.8 in 16 DCIS and IDC patients $(* *=P<0.005)$, unpaired t test. $\mathbf{f}$ Knockdown of the two target SE-IncRNAs was performed and expression of the SE-IncRNAs and their neighboring mRNAs was determined $48 \mathrm{~h}$ post-transfection in DCIS and CA1 cells, $\mathrm{n}=3,{ }^{*}=P<0.05$, paired t-test, error bars represent standard deviation

progression, but more importantly, our approach has identified 2 of the most promising, potentially cis-acting, target SE-lncRNAs (Fig. 4d, e) that, by regulating nearby gene expression, could be crucial in the progression of indolent DCIS to IDC.

\section{Classification of super-enhancers in breast cancer progression}

To complement our SE-lncRNA data, we identified super-enhancers in the MCF10A progression series. Enhancers are critical signaling elements regardless of 


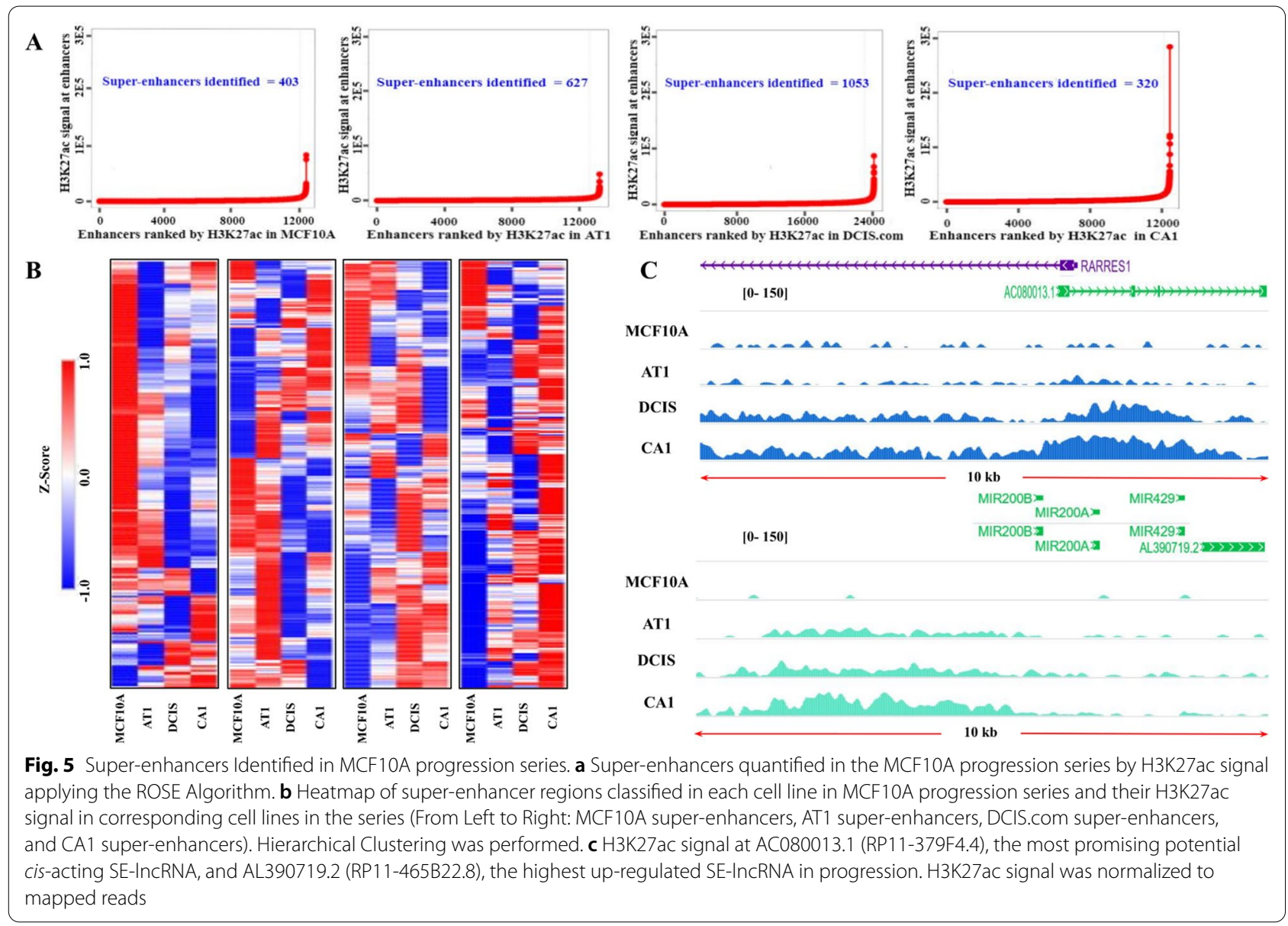

their association with SE-lncRNAs, therefore we performed H3K27ac chromatin immunoprecipitation (ChIP) to identify global enhancer activity (Additional file 3: Figure S3). This analysis enabled us to identify superenhancers at each stage of progression as well as active elements that may be predictive of progression. H3K27ac has previously been demonstrated to identify $2-3$ times more enhancer regions of interest than Med1 alone [33]. Super-enhancer regions were identified using the Rank Order Super-Enhancer (ROSE) algorithm [34] (Fig. 5a). Our analysis determined 403 super-enhancer regions in 10A, 627 in AT1, 1053 in DCIS, and 320 in CA1 cells (Additional file 8: Table S5). Interestingly, a stepwise increase in the number of super-enhancer regions was seen from MCF10As to DCIS cells, however in invasive CA1 cells the number of super-enhancers regions classified decreased. Although the number of classified superenhancers decreased in CA1 cells, the H3K signal is, on average, threefold more. In other words, even though the number of genomic regions that meet the threshold to be ranked as super-enhancers decrease from $10 \mathrm{~A}$ to CA1 cells, a higher H3K signal is seen in CA1 cells indicating a higher expression of those genomic regions.

Despite being classified as a super-enhancer in one cell line, a region may not be classified as a super-enhancer in another because ROSE compares signal intensity within cell lines and not between cell lines to rank superenhancers. Consequently, we analyzed H3K27ac signal intensity of super-enhancer regions identified in each cell line in the MCF10A progression series and observed their signal in the corresponding cell lines (Fig. 5b). Many of the super-enhancers classified in MCF10As lose signal intensity in AT1, DCIS, and CA1 cells indicating a loss of genomic expression within these regions. Similar trends of loss and gain of signal of the super-enhancers classified in AT1 and DCIS cells are seen (Fig. 5b). In conjunction with the higher H3K27ac signal observed for all CA1 identified super-enhancers, most of these regions are upregulated for H3K27ac signal (Fig. 5b). Correspondingly, we scanned the ranked super-enhancer regions in the progression series for our 2 identified SElncRNAs (Fig. 4d, e), RP11-379F4.4 and RP11-465B22.8. H3K27ac signal intensity was observed to increase in a 
stepwise manner for both targets (Fig. 5c), demonstrating that these genomic regions increase in accessibility in progression and contribute to a higher expression of their neighboring genes. Furthermore, RP11-379F4.4 is ranked as a super-enhancer in DCIS (\#8) as well as CA1 (\#92) cells (Additional file 8: Table S5). Though the region containing RP11-465B22.8 was not classified as a super-enhancer in our progression, it still exhibits higher H3K27ac occupancy in DCIS and CA1 cells when compared to normal. Specifically, the region upstream of RP11-465B22.8 and the miR-200 family is higher in H3K27ac signal intensity. Super-enhancer regions recruit transcription machinery and tend to loop to promoter regions of genes they regulate [35]. Hence, that region (and its H3K27ac occupancy) is consistent with the profile of an active enhancer involved in regulation of the expression of miR-200 family of genes with the help of RP11-465B22.8. These changes in the enhancer activation of DNA within progression are crucial to helping understanding progression of normal tissue to cancerous.

\section{Acquired/lost super-enhancers in breast cancer progression}

We examined our super-enhancer list to unravel newly acquired super-enhancers at each stage in progression in addition to super-enhancers lost at each stage relative to MCF10A cells (Fig. 6). 383 super-enhancers were newly acquired at the AT1 stage, 684 were newly acquired in DCIS, while only 28 were newly acquired at the CA1 stage (Additional file 8: Table S5). Consistent with previously established trends, most of the newly acquired super-enhancers were classified in AT1 and DCIS cells. Gene ontology assessment on the closest genes to these regions reveal many acquired pathways including STAT signaling in AT1 and NF-kB signaling in DCIS, which are putative pathways known for promoting proliferation and tumorigenesis (Fig. 6). Conversely, 173, 120, and 259 super-enhancers were lost at AT1, DCIS, and CA1 stages, respectively, (Additional file 8: Table S5). Similarly, a gene ontology assessment on genes (50 kb up or downstream (100 kb total) as enhancers tend to regulate genes within approximately a $50 \mathrm{~kb}$ distance upstream and downstream of its locus [29]) near lost super-enhancers reveal protein folding and local estrogen production as major pathways lost in progression (Fig. 6).

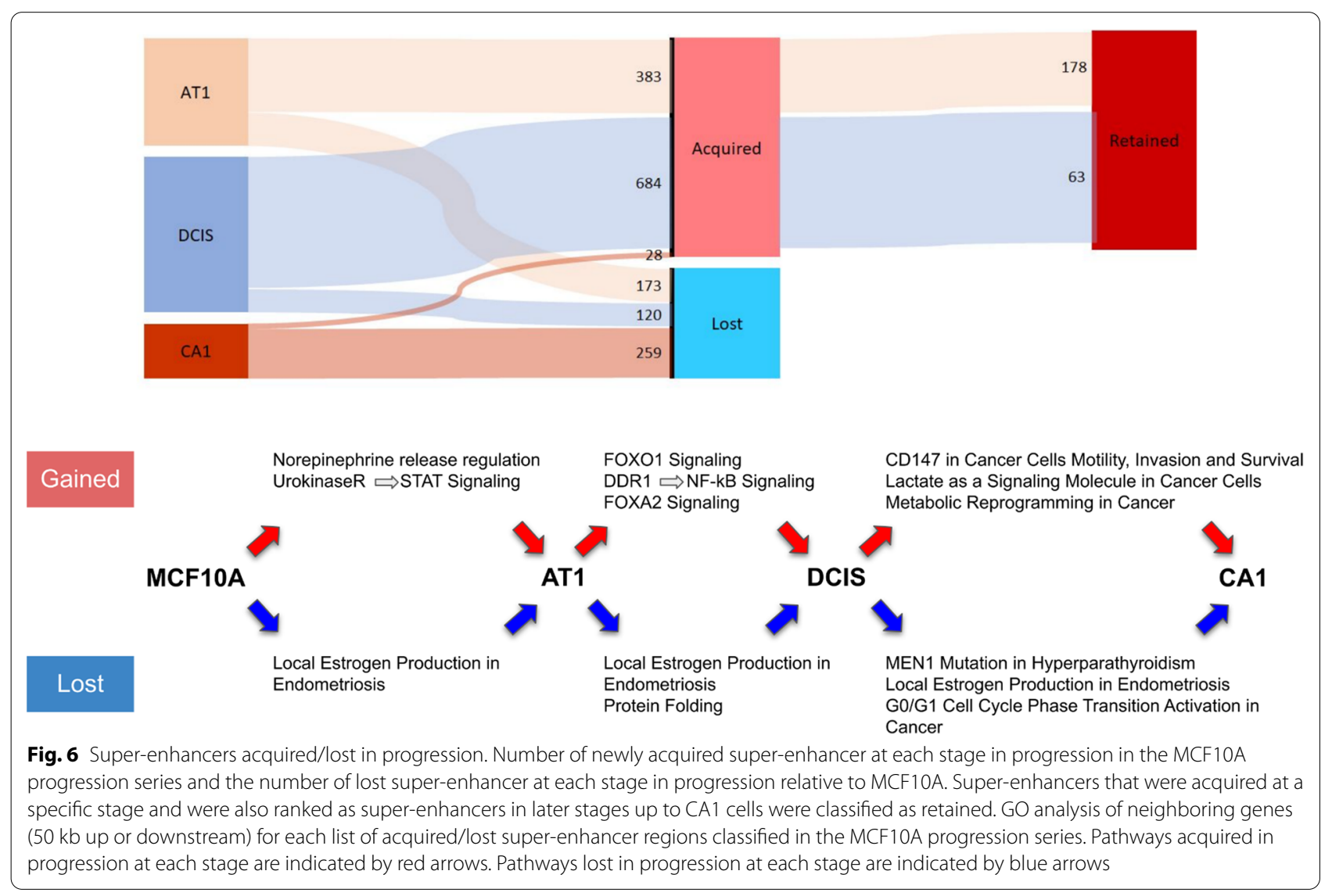


Comparison of acquired/lost super-enhancers in the MCF10A model with super-enhancers classified in ER + and triple negative breast cancer patients

To couple our MCF10A series findings with patient data, we identified super-enhancers in 47 estrogen receptor positive $(E R+)$ patient samples [36], 10 triple-negative breast cancer (TNBC) patient samples, and 11 triple-negative breast cancer cell lines (TNBCC) [37] (Additional file 9: Table S6). Comparison analysis of acquired superenhancer regions at each stage in progression was performed with super-enhancer regions in patient samples and TNBCC (Fig. 7a) (Additional file 9: Table S6). Superenhancers acquired/lost in progression that were most represented or absent in ER + and TNBC patients are listed with respect to their nearest gene (Fig. 7a). We highlight super-enhancer regions and their neighboring genes that were well represented in patient samples, TNBCC, and our progression series (Fig. 7b). Ephrin type-A receptor 2 (EphA2) region was classified as a super-enhancer at the DCIS stage in progression as well as being classified as a super-enhancer region in 34/47 ER+ patients and $10 / 10$ TNBC patients. Similarly, the region containing Cadherin 23 (CDH23) which was acquired at the CA1 stage in progression as a super-enhancer, was classified as a super-enhancer in 6/47 ER + patients and 10/10 TNBC patients. Interestingly, Transcription Factor AP-2 Alpha (TFAP2A) region was not ranked as a super-enhancer in any of the ER + patients while it was in all TNBC patients. Sumoylation of TFAP2A has been shown to block its ability to induce the expression of luminal genes and maintain a basal/triple-negative cancer subtype [38]. At the same time, genomic regions containing Glutaredoxin 2 (GLRX2), Laminin Subunit Alpha 2 (LAMA2), and Growth Arrest Specific 5 (GAS5), which are lost at the AT1 and DCIS stages, respectively, were not ranked as super-enhancers in any of the patients or TNBC cell lines. GAS5 is a well-studied down regulated lncRNA in breast cancer while GLRX2 is a protein that localizes to the mitochondria where it functions in mitochondrial redox homeostasis and is important for the protection against and recovery from oxidative stress [39]. Considering metabolic reprogramming is one of the pathways acquired (Fig. 6), epigenetic changes within the GLRX2 region could play a crucial role in progression. Super-enhancers are central to driving expression of genes controlling cell identity and stimulating oncogenic

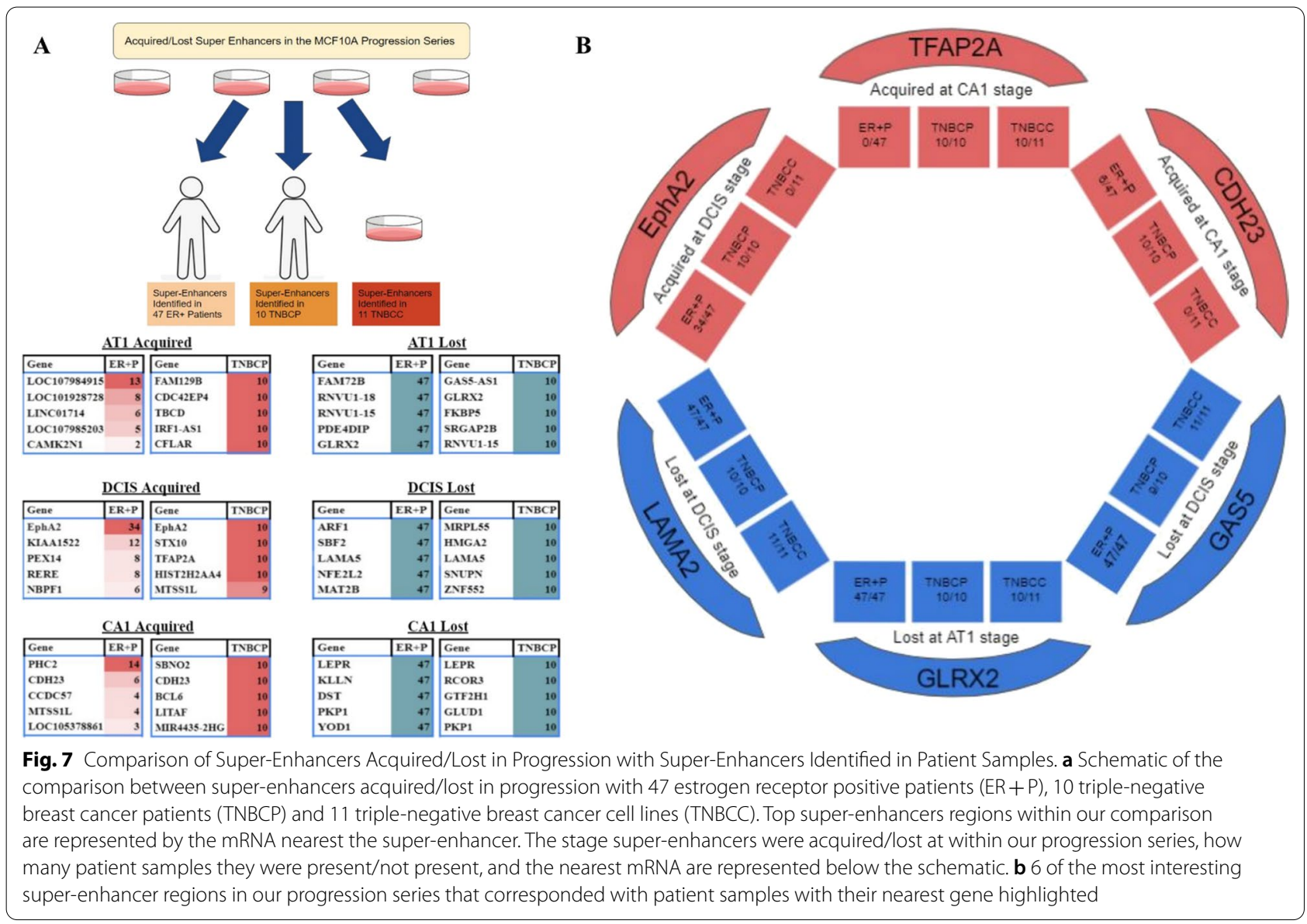


transcription, thus, cancerous phenotype relies on these abnormal transcription propelled by super-enhancers. Here we have highlighted super-enhancers acquired/ lost in progression and cross-referencing these regions with super-enhancers in patient samples, we unravel epigenetic changes driving cell identity and progression. Lastly, this validates results from our model with patient samples and current literature demonstrating a robust discovery platform.

\section{Discussion}

Currently, functional determinants of DCIS progression to an invasive lesion are unknown[17]. This study profiles global SE-lncRNA expression in the MCF10A progression series giving insight into numerous SE-lncRNAs that are differentially expressed in progression. Furthermore, these SE-lncRNAs can play essential roles in transcriptional regulation through controlling SEs activity to regulate a broad range of physiological and pathological processes, especially tumorigenesis. Equally, SE-lncRNAs can regulate gene expression by affecting gene promoter activity. Although SE-lncRNAs significantly contribute to gene expression, the systematic identification of SE-lncRNAs and their regulated genes still lacks comprehensive recognition [40] [41]. Hence, this study also elucidates the expression levels of mRNAs associated with those SElncRNAs. In addition, using stringent and comprehensive set of filters that combined Cancer Cell line data with patient data, we have highlighted 27 potentially cis-acting SE-IncRNAs and their target mRNAs coupled with 4 SElncRNAs that are highest differentiated in disease progression. From this list we have identified RP11-379F4.4 (AC080013.1) as a promising cis-acting SE-lncRNA to its target gene Retinoic acid receptor responder element 1 (RARRES1). Interestingly, RARRES1 functions as an invasion suppressor. This function of the gene was confirmed in metastatic prostate cancer $(\mathrm{CaP})$ cell line (PC3M) by Oldridge et al. [42]. Likewise, RARRES1 is able to increase Sirtuin 1, while it decreases the mechanistic target of rapamycin (mTOR), two important regulators of energy homeostasis. RARRES1 is differentially expressed in metabolic diseases and is associated with biological hallmarks that require metabolic reprogramming. Metabolic reprogramming is now considered a hallmark of cancer etiology [43]. Although RARRES1 is among the most commonly methylated genes in multiple cancers, it is increased in basal-like hormone receptor negative breast cancer and in liver cirrhosis, a risk factor for hepatocellular cancer [44].

Similarly, we also have highlighted RP11-465B22.8 (AL390712.1) as the most differentiated SE-lncRNA in progression. Comparatively, miR-200b, neighboring RP11-465B22.8, is part of the well-known tumor suppressor miR200 family. The family of miR-200 includes five members: miR-200a, miR-200b, miR200c, miR-429, and miR-141. miR-200b, which acts as an antioncogene, participates in the proliferation and metastasis inhibition of different kinds of cancers by downregulating target molecules. For instance, miR$200 \mathrm{~b}$ inhibition promotes Rac1 activation and increases the metastatic potential of HBEC cells [45]. miR-200b can repress angiogenesis by targeting angiogenic factors and receptors [46]. It can inhibit the epithelial to mesenchymal transition (EMT) by inactivating transcription factors in breast cancer. miR-200b is associated with the estrogen receptor status of breast cancer cells [46, 47]. Zheng et al. also highlight Fucosyltransferase IV (FUT4) could apply as a novel target for miR-200b that suppresses the proliferation and metastasis of breast cancer cells by reducing $\alpha 1,3$-fucosylation and LeY biosynthesis of glycoproteins [31]. Thus, SE-lncRNAs RP11-379F4.4 and RP11-465B22.8 and their respective potential targets are promising candidates for their cis-acting capabilities leading to progression. Further studies will have to be conducted to assess the mechanism of cis action for the SE-lncRNAs and how they promote DCIS lesions toward IDC.

In the past decade, increasing evidence has revealed that super-enhancers play a vital role in tumorigenesis and there is great interest in developing super-enhancer therapeutics, thus, this study also elucidates superenhancers that are acquired or lost in progression [6]. We profiled H3K27ac using ChIP in the MCF10A progression series. As enhancers and super-enhancers play an important role in driving cell identity, the alteration in intensity of the $\mathrm{H} 3 \mathrm{~K} 27 \mathrm{ac}$ in progression paints a picture of epigenetic changes which could be leading to the hijacking of genes involved in various hallmarks of cancer.

Correspondingly, we uncover newly acquired superenhancers at each stage in progression in addition to super-enhancers lost at each stage relative to MCF10A cells. This analysis enabled us to highlight and distinguish loci that are activated/suppressed. Importantly, we identified 28 super-enhancers that are acquired from DCIS to CA1 transition and over 100 super-enhancers that are lost from DCIS to CA1. Furthermore, we classify genes neighboring these acquired/lost regions which identify pathways that contribute to progression. For example, STAT signaling is acquired in AT1 transition from normal cells, while NF-kB signaling is acquired in the transition to DCIS. Similarly, protein folding and local estrogen production pathways are lost overall in progression in addition to regions responsible for cell cycle regulation. These are canonical cancer pathways leading to proliferation and metastasis. Here we show how 
super-enhancers being activated/suppressed neighboring genes involved in these pathways play a pivotal role in their misregulation.

Comparison of acquired/lost super-enhancer regions with super-enhancer regions classified in 47 $\mathrm{ER}+$ patients, $10 \mathrm{TNBC}$ patients, and $11 \mathrm{TNBC}$ cell lines provides clinical relevance. This comprehensive analysis reveals epigenetic changes at the genome wide level in breast tumors. For example, Ephrin type-A receptor 2 (EphA2), is a receptor tyrosine kinase which binds ephrin-A family ligands residing on adjacent cells, leading to contact-dependent bidirectional signaling into neighboring cells. This gene has been known to regulate migration, integrin-mediated adhesion, proliferation, and differentiation of cells through DSG1/desmoglein-1 and inhibition of the ERK1/ERK2 signaling pathway [48]. EphA2 has been implicated in breast tumors and resistance of tumors to targeted therapies [48-50]. Targeting EphA2 has been shown to inhibit cell cycle progression and proliferation [48]. The genomic region containing EphA2 is an acquired super-enhancer at the DCIS stage in our progression model. Interestingly, it is also classified as a super-enhancer region in 34/47 ER + patient samples and 10/10 TNBC patients in our analysis. This explains the observed overexpression of this gene in breast tumors and can provide new targeting methods. Identically, the region containing GAS5 IncRNA is classified as a superenhancer on normal MCF10A cells, however, is lost at the AT1, DCIS, and CA1 stages. Neither is it classified as a super-enhancer in all 47 ER + and 9/10 TNBC patients implying a mechanism of the down-regulation seen of this lncRNA in breast tumors. GAS5 can bind to the DNA binding domain of the glucocorticoid receptor inactivating it and subsequently inhibiting the regulation of its target genes [51]. In addition, GAS5 can regulate the transcriptional activity of other receptors, such as androgen and progesterone and has been suggested as a potential tumor suppressor due to its pro-apoptotic function [52]. The epigenetic changes occurring within this region can explain the down-regulation of this lncRNA and reduce its effects in carrying out normal processes leading to tumorigenesis.

Lastly, we also analyzed the regions that contained our two identified targets, RP11-379F4.4 and RP11-465B22.8, to see if they were classified as super-enhancers in progression. RP11-379F4.4 was ranked as a super-enhancer in DCIS as well as CA1 cells and is seen to be acquired during progression. RP11-465B22.8 was not ranked as a super-enhancer by the ROSE algorithm in any of the cells, however, the signal intensity of H3K27ac saw a dramatic stepwise increase from normal to CA1 cells. This result verifies the enhanced transcription that is observed of these SE-lncRNAs and hints at a possible role that they may play to induce progression. We also examined if RP11-379F4.4, which was an acquired super-enhancer at the DCIS stage in progression, was classified as a superenhancer in any of the patient samples. Although RP11379F4.4 was not classified as a super-enhancer in patient samples, its classification as an enhancer represents open DNA, and most likely, the cause of its higher expression seen in CA1 cells and patient tumors. Super-enhancers associate with key oncogenes in cancers and drive expression of genes that define cell identity. Additionally, cancer cells can acquire super-enhancers at oncogenes while losing super-enhancers at tumor suppressing genes. Understanding the alterations in the genomic landscape within breast tumors will uncover underlying biology that can be useful in diagnostic and targeted therapies.

The data presented here highlight several SE-IncRNAs that through their potential cis-acting abilities play an important role in progression of DCIS lesions into invasive IDC. Furthermore, we have identified two promising target SE-lncRNAs that may drive cancer progression through the regulation of their neighboring gene. Future studies will expand on understanding their potential cisacting functions. Equally, we reveal acquired/lost superenhancers in progression coupled with patient data that can help elucidate the epigenetic alterations promoting cancerous phenotypes. While there may not be one key protein that determines DCIS progression, understanding the networks of signaling pathways that change during progression unravel critical changes that push a DCIS lesion to be invasive.

\section{Conclusion}

Altogether, this comprehensive study of breast cancer cell lines coupled with patient samples provides a unique platform that identifies differentially expressed SE-lncRNAs and acquired/lost super-enhancers in progression of breast cancer important for promoting DCIS lesions to IDC.

\section{Abbreviations \\ DCIS: Ductal carcinoma in situ; IDC: Invasive ductal carcinoma; SE: Super- enhancer; e-IncRNA: Enhancer long non-coding RNA; SE-IncRNA: Super- enhancer long non-coding RNA; 10A: MCF10A; AT1: MCF10A-AT1; DCIS: MCF10A-DCIS; CA1: MCF10A-CA1; ChIP: Chromatin immunoprecipitation; ASO: Antisense oligonucleotide; ER + : Estrogen receptor positive; TNBC: Triple negative breast cancer.}

\section{Supplementary Information}

The online version contains supplementary material available at https://doi. org/10.1186/s13058-021-01479-8.

Additional file 1: Figure S1: Localization of the potential cis-acting SEIncRNAs. Localization of the 12 SE-IncRNAs from our list of 27 potentially 
cis-acting SE-IncRNAs and 4 highest differentiated that are primarily localized within the cytoplasm. Act-b and $18 \mathrm{~s}$ are used as controls.

Additional file 2: Figure S2: Expression levels of potential cis-acting SE-IncRNAs in breast cancer patients. A) Expression levels of remaining up-regulated SE-InCRNAs in $24 \mathrm{DCIS}$ and 24 IDC patients, ${ }^{*}=\mathrm{P}<0.05$. B) Expression levels of remaining down-regulated SE-IncRNAs in 24 DCIS and 24 IDC patients, ${ }^{*}=P<0.05$. RP11-61F12 does show a statistically significant increase in expression from DCIS to IDC in patient samples, and this mimics our progressions series data. It has been marked for future studies; however, it is not the most promising of our target SE-IncRNAs.

Additional file 3: Figure S3: Specificity of H3K27ac ChIP antibody. Fold enrichment of the H3K27ac antibody between control promoter region of Myt1 and enhancer region of MYC.

Additional file 4: Table S1: Raw values of all SE-InCRNAs in the MCF10A progression series.

Additional file 5: Table S2: 138 SE-IncRNAs, their corresponding mRNAs, and the $\log 2$ fold change of each in the MCF10A progression series.

Additional file 6: Table S3: 27 SE-IncRNAs, their associated gene, and all mRNAs correlated within the TANRIC database with their Pearson Correlation Coefficient that met our threshold.

Additional file 7: Table S4: Statistical significance for SE-InCRNAs and their associated mRNAs expression in our progression series characterized by one-way ANOVA with Tukey correction. All significant and non-significant results are shown.

Additional file 8: Table S5: Super-enhancers identified using H3K27ac ChIP seq and classified by the ROSE algorithm in each cell line in the MCF10A progression series.

Additional file 9: Table S6: Super-enhancers identified in 47 ER+, 10 TNBC, and 11 TNBCC compared with super-enhancers identified in each cell line in the MCF10A progression series.

\section{Acknowledgements}

Special thanks to Marcy Kuentzel and Andrew Hayden at the CFG Core SUNY Albany for their expertise and help with ChIP sequencing, qPCR expression data, and patient data.

\section{Authors' contributions}

Ali S. Ropri-designed the study, performed all experiments and analysis, and wrote the paper. Rebecca DeVaux — designed the study, analyzed microarray data, helped with patient expression data and analysis. Jonah Eng — performed ROSE analysis of ER + and TNBC patient samples, performed statistical analysis of in-house patient samples. Sridar V. Chittur-provided expertise on sequencing of ChIP seq samples and helped with ChIP seq experiments. Jason Herschkowitz - principal investigator and mentor. All authors read and approved the final manuscript.

\section{Funding}

This work was supported by Susan G Komen Foundation Grant 1144184-1-80226.

\section{Availability of data and materials}

The dataset generated and analyzed during the current study is available from the corresponding author on request. In addition, the H3K27ac ChIP-seq data for the MCF10A progression series discussed in this publication has been deposited in NCBI's Gene Expression Omnibus database under accession number GSE181524.

\section{Declarations}

\section{Ethics approval and consent to participate}

The protocol of deriving primary human epithelial cells from normal tissues adjacent to breast tumors from breast cancer patients was approved by the institutional review board at Fox Chase Cancer Center.

\section{Consent for publication}

Not applicable.

\section{Competing interests}

The authors declare no competing interests.

\section{Author details}

${ }^{1}$ Department of Biomedical Sciences, Cancer Research Center, University at Albany, 1 Discovery Drive, Suite 317, Rensselaer, NY 12144, USA. ${ }^{2}$ Bethlehem Central High School, Bethlehem Central School District, Delmar, NY 12054, USA. ${ }^{3}$ Center for Functional Genomics, Cancer Research Center, University at Albany, Rensselaer, NY 12144, USA.

Received: 10 August 2021 Accepted: 20 October 2021

Published online: 30 October 2021

\section{References}

1. Vaidya JC, Patkar V. Fast facts: early. Breast Cancer. 2016. https://doi.org/ 10.1159/isbn.978-1-910797-25-9.

2. Breast Cancer Facts \& Figures. https://www.cancer.org/research/cancerfacts-statistics/breast-cancer-facts-figures.html. Accessed 3 Sep 2020

3. Lian J, Li K. A review of breast density implications and breast cancer screening. Clin Breast Cancer. 2020. https://doi.org/10.1016/j.clbc.2020.03. 004.

4. Virnig BA, Wang S-Y, Shamilyan T, Kane RL, Tuttle TM. Ductal carcinoma in situ: risk factors and impact of screening. JNCI Monogr. 2010;2010:113-6.

5. Mansour MR, Abraham BJ, Anders L, et al. An oncogenic super-enhancer formed through somatic mutation of a noncoding intergenic element. Science. 2014;346:1373-7.

6. pubmeddev, Thandapani P Super-enhancers in cancer. - PubMed - NCBI. https://www.ncbi.nlm.nih.gov/pubmed/30885876. Accessed 18 Apr 2020

7. Tang F, Yang Z, Tan Y, Li Y. Super-enhancer function and its application in cancer targeted therapy. NPJ Precis Oncol. 2020;4:2.

8. He Y, Long W, Liu Q. Targeting super-enhancers as a therapeutic strategy for cancer treatment. Front Pharmacol. 2019. https://doi.org/10.3389/ fphar.2019.00361.

9. Gregg C (2013) Faculty of 1000 evaluation for super-enhancers in the control of cell identity and disease. F1000_-post-publication peer review of the biomedical literature. https://doi.org/10.3410/f.718140971.79348 6542

10. Hnisz D, Abraham BJ, Lee TI, Lau A, Saint-André V, Sigova AA, Hoke HA, Young RA. Super-enhancers in the control of cell identity and disease. Cell. 2013;155:934-47.

11. Hou Y, Zhang R, Sun X. Enhancer LncRNAs influence chromatin interactions in different ways. Front Genet. 2019. https://doi.org/10.3389/fgene. 2019.00936.

12. Rao MRS. Long non-coding RNA biology. Berlin: Springer; 2017.

13. Factor DC, Tesar PJ, Khalil AM (2013) Chromatin regulation by long noncoding RNAs. Molecular Biology of Long Non-coding RNAs, 1-13

14. Wang KC, Yang YW, Liu B, et al. A long noncoding RNA maintains active chromatin to coordinate homeotic gene expression. Nature. 2011:472:120-4.

15. Sigova AA E al Transcription factor trapping by RNA in gene regulatory elements.—PubMed—NCBI. https://www.ncbi.nlm.nih.gov/pubmed/ 26516199. Accessed 18 Apr 2020

16. Lee J-H, Xiong F, Li W (2020) Enhancer RNAs in cancer: regulation, mechanisms, and therapeutic potential. RNA Biol, pp. 1-10

17. DeVaux RS, Ropri AS, Grimm SL, Hall PA, Herrera EO, Chittur SV, Smith WP, Coarfa C, Behbod F, Herschkowitz Jl. Long noncoding RNA BHLHE40-AS1 promotes early breast cancer progression through modulating IL-6/STAT3 signaling. J Cell Biochem. 2020;121:3465-78.

18. Nguyen TA, Jones RD, Snavely AR, Pfenning AR, Kirchner R, Hemberg $M$, Gray JM. High-throughput functional comparison of promoter and enhancer activities. Genome Res. 2016;26:1023-33.

19. Ernst J, Melnikov A, Zhang X, Wang L, Rogov P, Mikkelsen TS, Kellis M. Genome-scale high-resolution mapping of activating and repressive nucleotides in regulatory regions. Nat Biotechnol. 2016;34:1180-90. 
20. Soule HD, Maloney TM, Wolman SR, Peterson WD Jr, Brenz R, McGrath CM, Russo J, Pauley RJ, Jones RF, Brooks SC. Isolation and characterization of a spontaneously immortalized human breast epithelial cell line, MCF-10. Cancer Res. 1990;50:6075-86.

21. Miller FR, Santner SJ, Tait L, Dawson PJ. MCF10DCIS.com xenograft model of human comedo ductal carcinoma in situ. J Natl Cancer Inst. 2000;92:1185a-186.

22. Santner SJ, Dawson PJ, Tait L, Soule HD, Eliason J, Mohamed AN, Wolman SR, Heppner GH, Miller FR. Malignant MCF10CA1 cell lines derived from premalignant human breast epithelial MCF10AT cells. Breast Cancer Res Treat. 2001;65:101-10.

23. Shi Y, Shang J (2016) Long noncoding RNA expression profiling using arraystar LncRNA microarrays. Long Non-Coding RNAs, pp 43-61

24. Super-enhancer IncRNA Array Service. https://www.arraystar.com/superenhancer-Incrna-array-service/. Accessed 27 May 2021

25. Gil N, Ulitsky I. Regulation of gene expression by cis-acting long noncoding RNAs. Nat Rev Genet. 2020;21:102-17.

26. Hezroni H, Koppstein D, Schwartz MG, Avrutin A, Bartel DP, Ulitsky I. Principles of long noncoding RNA evolution derived from direct comparison of transcriptomes in 17 species. Cell Rep. 2015;11:1110-22.

27. Derrien T, Johnson R, Bussotti G, et al. The GENCODE v7 catalog of human long noncoding RNAs: analysis of their gene structure, evolution, and expression. Genome Res. 2012;22:1775-89.

28. Ong C-T, Corces VG. Enhancer function: new insights into the regulation of tissue-specific gene expression. Nat Rev Genet. 2011;12:283-93.

29. Chepelev I, Wei G, Wangsa D, Tang Q, Zhao K. Characterization of genome-wide enhancer-promoter interactions reveals co-expression of interacting genes and modes of higher order chromatin organization. Cell Res. 2012;22:490-503.

30. Li J, Han L, Roebuck P, Diao L, Liu L, Yuan Y, Weinstein JN, Liang H. TANRIC: an interactive open platform to explore the function of IncRNAs in cancer. Cancer Res. 2015;75:3728-37.

31. Zheng Q, Cui X, Zhang D, et al. (2017) miR-200b inhibits proliferation and metastasis of breast cancer by targeting fucosyltransferase IV and a1,3fucosylated glycans. Oncogenesis 6:e358

32. Sánchez-Cid L, Pons M, Lozano JJ, et al. MicroRNA-200, associated with metastatic breast cancer, promotes traits of mammary luminal progenitor cells. Oncotarget. 2017;8:83384-406.

33. Fong PK, Lee NK (2015) Improved H3K27ac histone mark prediction using k-mer proximity feature. In: 2015 9th International Conference on IT in Asia (CITA). https://doi.org/10.1109/cita.2015.7349830

34. Whyte WA, Orlando DA, Hnisz D, Abraham BJ, Lin CY, Kagey MH, Rahl PB, Lee TI, Young RA. Master transcription factors and mediator establish super-enhancers at key cell identity genes. Cell. 2013;153:307-19.

35. Hsieh C-L, Fei T, Chen Y, et al. Enhancer RNAs participate in androgen receptor-driven looping that selectively enhances gene activation. Proc Natl Acad Sci U S A. 2014;111:7319-24.

36. Patten DK, Corleone G, Győrffy B, et al. Enhancer mapping uncovers phenotypic heterogeneity and evolution in patients with luminal breast cancer. Nat Med. 2018;24:1469-80.

37. Raisner R, Bainer R, Haverty PM, Benedetti KL, Gascoigne KE (2020) Superenhancer acquisition drives oncogene expression in triple negative breast cancer. PLoS ONE 15:e0235343

38. Bogachek MV, Chen Y, Kulak MV, Woodfield GW, Cyr AR, Park JM, Spanheimer PM, Li Y, Li T, Weigel RJ. Sumoylation pathway is required to maintain the basal breast cancer subtype. Cancer Cell. 2014;25:748-61.
39. Gladyshev VN, Liu A, Novoselov SV, Krysan K, Sun Q-A, Kryukov VM, Kryukov GV, Lou MF. Identification and characterization of a New Mammalian Glutaredoxin (Thioltransferase), Grx2. J Biol Chem. 2001;276:30374-80.

40. McKinsey T (2018) Faculty Opinions recommendation of Transcription of the non-coding RNA upperhand controls Hand2 expression and heart development. Faculty Opinions-Post-Publication Peer Review of the Biomedical Literature. https://doi.org/10.3410/f.726887070.793551660

41. Ounzain S, Micheletti R, Arnan C, et al. CARMEN, a human super enhancer-associated long noncoding RNA controlling cardiac specification, differentiation, and homeostasis. J Mol Cell Cardiol. 2015;89:98-112.

42. Oldridge EE, Walker HF, Stower MJ, Simms MS, Mann VM, Collins AT, Pellacani D, Maitland NJ (2013) Retinoic acid represses invasion and stem cell phenotype by induction of the metastasis suppressors RARRES1 and LXN. Oncogenesis 2:e45

43. Pavlova NN, Thompson CB. The emerging hallmarks of cancer metabolism. Cell Metab. 2016;23:27-47.

44. Maimouni S, Issa N, Cheng S, Ouaari C, Cheema A, Kumar D, Byers S (2018) Tumor suppressor RARRES1 - a novel regulator of fatty acid metabolism in epithelial cells. PLoS ONE 13:e0208756

45. Wang Z, Humphries B, Xiao H, Jiang Y, Yang C. MicroRNA-200b suppresses arsenic-transformed cell migration by targeting protein kinase $\mathrm{Ca}$ and Wnt5b-protein kinase Ca positive feedback loop and inhibiting Rac1 activation. J Biol Chem. 2014;289:18373-86.

46. Chang $S-H, L$ L Y $-C, L i X$, Hsieh W-Y, Xiong Y, Ghosh M, Evans T, Elemento $\mathrm{O}$, Hla T. Antagonistic function of the RNA-binding protein HuR and miR$200 \mathrm{~b}$ in post-transcriptional regulation of vascular endothelial growth factor-a expression and angiogenesis. J Biol Chem. 2013;288:4908-21.

47. Gregory PA, Bert AG, Paterson EL, Barry SC, Tsykin A, Farshid G, Vadas MA, Khew-Goodall Y, Goodall GJ. The miR-200 family and miR-205 regulate epithelial to mesenchymal transition by targeting ZEB1 and SIP1. Nat Cell Biol. 2008;10:593-601.

48. Song W, Hwang Y, Youngblood VM, Cook RS, Balko JM, Chen J, BrantleySieders DM. Targeting EphA2 impairs cell cycle progression and growth of basal-like/triple-negative breast cancers. Oncogene. 2017;36:5620-30.

49. Tandon M, Vemula SV, Mittal SK. Emerging strategies for EphA2 receptor targeting for cancer therapeutics. Exp Opin Ther Targets. 2011;15:31-51.

50. Martini G, Cardone C, Vitiello PP, et al. EPHA2 is a predictive biomarker of resistance and a potential therapeutic target for improving antiepidermal growth factor receptor therapy in colorectal cancer. Mol Cancer Ther. 2019;18:845-55.

51. Ni W, Yao S, Zhou Y, Liu Y, Huang P, Zhou A, Liu J, Che L, Li J. Long noncoding RNA GAS5 inhibits progression of colorectal cancer by interacting with and triggering YAP phosphorylation and degradation and is negatively regulated by the m6A reader YTHDF3. Mol Cancer. 2019. https:// doi.org/10.1186/s12943-019-1079-y.

52. Kino T, Hurt DE, Ichijo T, Nader N, Chrousos GP (2010) Noncoding RNA gas 5 is a growth arrest- and starvation-associated repressor of the glucocorticoid receptor. Sci Signal 3:ra8

\section{Publisher's Note}

Springer Nature remains neutral with regard to jurisdictional claims in published maps and institutional affiliations.
Ready to submit your research? Choose BMC and benefit from:
- fast, convenient online submission
- thorough peer review by experienced researchers in your field
- rapid publication on acceptance
- support for research data, including large and complex data types
- gold Open Access which fosters wider collaboration and increased citations
- maximum visibility for your research: over $100 \mathrm{M}$ website views per year

At $\mathrm{BMC}$, research is always in progress.

Learn more biomedcentral.com/submissions 